\title{
Genomic epidemiological models describe pathogen evolution across fitness valleys
}

Preprint deposited to biorXiv on 16 December, 2021

\section{Authors:}

Pablo Cárdenas ${ }^{1} \&$ Mauricio Santos-Vega ${ }^{2}$

\author{
Affiliations: \\ ${ }^{1}$ Department of Biological Engineering, Massachusetts Institute of Technology, Cambridge, MA \\ 02139, USA. Correspondence: pcarden@mit.edu \\ ${ }^{2}$ Grupo Biología Matemática y Computacional, Departamento Ingeniería Biomédica, \\ Universidad de los Andes, Bogotá, D.C. 111711, Colombia.
}

\begin{abstract}
:
Genomics is fundamentally changing epidemiological research. However, exploring hypotheses about pathogen evolution in different epidemiological contexts poses new challenges. Models intertwining pathogen epidemiology and genomic evolution can help understand processes such as the emergence of novel pathogen genotypes with higher transmission or resistance to treatment. In this work, we present Opqua, a computational framework for flexible simulation of pathogen epidemiology and evolution. We use Opqua to study determinants of evolution across fitness valleys. We confirm that competition can limit evolution in high transmission environments and find that low transmission, host mobility, and complex pathogen life cycles facilitate reaching new adaptive peaks through population bottlenecks and decoupling selective pressures. The results show the potential of genomic epidemiological modeling as a tool in infectious disease research.
\end{abstract}

\section{Introduction}

Genomic epidemiology has become a powerful tool in the study and control of infectious disease spread. By sequencing the genomes of pathogens in the field, researchers can access a living ledger of transmission and evolution in real-time, which aids understanding of disease spread and can inform public health policy. Sequencing of pathogen genomes has been used to 
monitor evolution, trace local chains of transmission, and study the origins of outbreaks in real-time for Ebola (1-3), malaria (4-6), influenza (7, 8), and COVID-19 (9-19), among others. An arsenal of tools and initiatives have been developed to harness the full potential of data generated through genomic surveillance $(4,20-23)$.

Despite all its advantages, genomic epidemiology is hampered by its ability to experimentally explore hypotheses beyond observational data in the same way most epidemiological research is. Fortunately, mathematical and computational modeling provide frameworks for investigating the effect of individual variables through the simulation of null models and perfectly-controlled, targeted interventions, which would otherwise be impossible in practice $(12,24-26)$. However, most traditional modeling frameworks are not well-suited to work with genomic data and the kinds of evolutionary questions unlocked by genomic epidemiology. For example, different computational tools have been developed to simulate the evolution of pathogen genetic sequences (27-29), but these tools are not coupled to epidemiological models that follow disease spread.

More recently, there has been interest in developing tools to combine epidemiological models with sequence evolution simulators [such as mrc-ide.github.io/SIMPLEGEN/index.html, (30-32)]. While these approaches can be powerful tools to answer questions about population genetics, they do not consider the effect of genetic sequences on disease dynamics. They thus are unable to account for selection in pathogen evolution. More general tools are available to simulate the evolution of organism populations in forward-time (33-35). Although these may be adapted to use in epidemiology, this requires significant work and setup, especially when tailoring to different kinds of disease transmission and intervention. More recent work has been done to explore evolving epidemiological models in a custom, disease-specific context (36). Nevertheless, there currently is no out-of-the-box solution for building flexible, easy-to-use simulations of disease spread with pathogens capable of evolving and influencing their epidemiology.

Simulating selection and evolution becomes particularly pressing as we ask questions about how epidemiology affects the appearance of advantageous traits within a pathogen population. Specifically, we are interested in how epidemiological contexts can shape competition and clonal interference between pathogens to hamper the evolution of novel traits separated by fitness valleys. Significant work has examined the evolutionary dynamics of crossing fitness valleys, a process termed stochastic tunneling (37-39). Other work has examined the environmental factors that shape these adaptive landscapes (40). Still, quantitative research into stochastic tunneling in the context of a spreading infection has only recently become an object of study (36). This is notable given the interest in the evolutionary biology of infectious 
diseases sparked by the COVID-19 pandemic. As SARS-CoV-2 variants approach semi-stable, local peaks in fitness (41), the role of epidemiology in shaping evolutionary trajectories across fitness valleys becomes crucial in understanding future viral evolution and vaccine effectiveness. The emergence of novel, evolutionarily distant SARS-CoV-2 variants such as Omicron is a concerning testament to the importance of these questions, as this new variant bears multiple compensatory mutations and epistatic interactions that individually decrease fitness, but have a synergistic effect on transmission $(42,43)$. A study of the eco-epidemiological determinants of evolution is also key to understanding the emergence of drug resistance in diseases such as tuberculosis and malaria, where fitness costs of resistant mutations are offset by a succession of compensatory mutations $(44,45)$.

In light of this, we developed Opqua (github.com/pablocarderam/opqua) as an epidemiological modeling framework for pathogen population genetics and evolution. We apply Opqua to explore how different aspects of the epidemiological environment and infection biology constrain or enhance the ability of pathogens to escape local fitness peaks and explore new evolutionary space in adaptive landscapes.

\section{Results}

A computational modeling framework for genomic epidemiology and evolution

In order to address questions in infectious disease evolution, we developed a library for flexible epidemiological simulations of evolving pathogens. The library is named Opqua, after the word for disease, cause, or reason in the Chibcha family of languages spoken by members of the late Muysca Confederation in modern-day Colombia (46). 

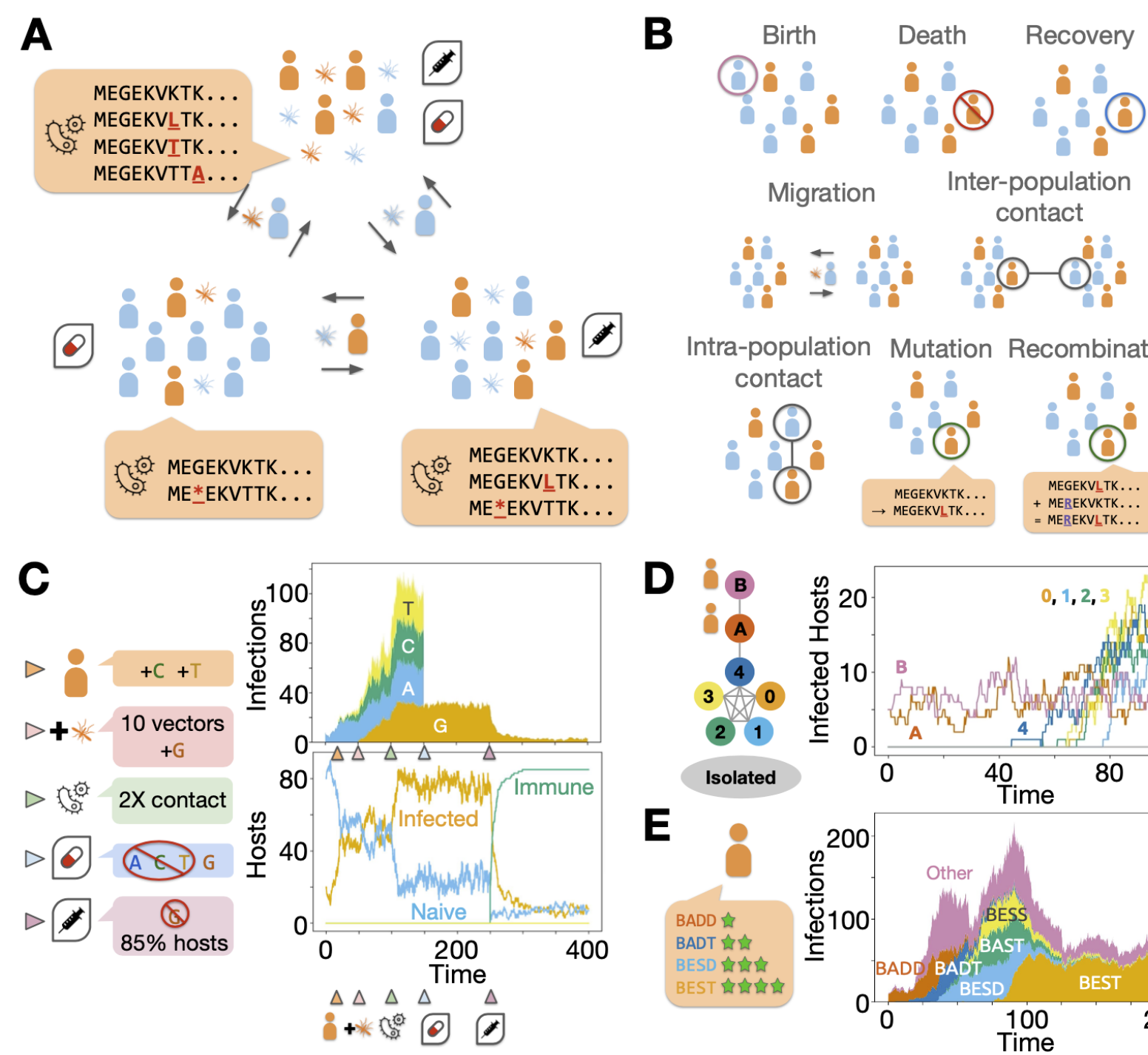

Intra-population Mutation Recombination
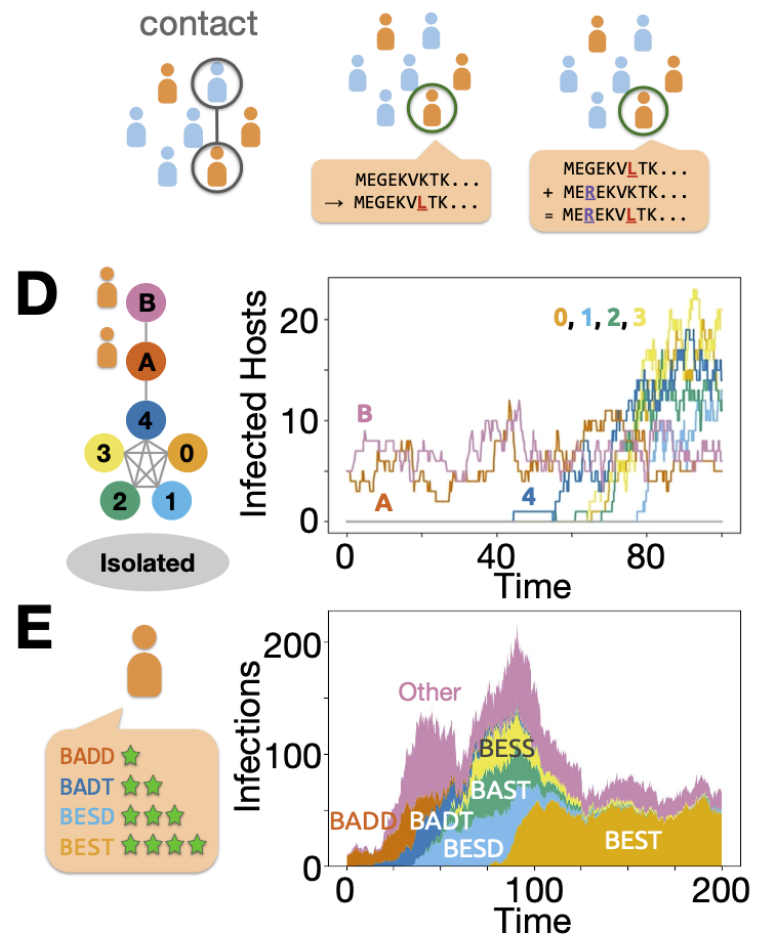

Fig. 1. Opqua simulates stochastic, structured epidemiological models of evolving pathogens. (A) Opqua models account for pathogens with arbitrary genomes and genomic alphabets spreading through structured populations of hosts and/or vectors, which may acquire immunity and/or undergo interventions such as drug treatment or vaccination. (B) Eight possible kinds of events can occur in simulations, the rates of which may be influenced by pathogen genome sequence. (C) Models may include interventions at different moments in time, which in this example include addition of new hosts and vectors infected with pathogens of specific genome sequences, altering epidemiological conditions to increase host-vector contact rate, administration of a treatment that kills all pathogens except those with a specific, resistant genotype, and finally, vaccination against the same genotype. (D) Models may have arbitrary, complex metapopulation structures, here showing the spread of disease from two low-transmission populations ( $A$ " and " $B$ ") into five interconnected populations with high transmission (labeled 1-5), while an additional isolated population remains disease-free. (E) Models can simulate evolution and selection through different ways, here showing a population of pathogens that evolve through direct intra-host competition and de novo mutation from an initial, low-fitness genotype ("BADD") to a high-fitness, optimal genotype ("BEST"). 
Briefly, Opqua stochastically simulates interconnected populations of agents representing hosts and/or vectors, which may be infected with pathogens (Fig. 1A). Simulations occur through different kinds of demographic, epidemiological, and genomic events that affect the state of the system, and thus the probability of the next event (Fig. 1B; Fig. S1). User-defined epidemiological parameters affect the rate of each event and can vary both across populations and throughout the course of each simulation, allowing the user to model different ecological events or public health interventions (Fig. 1C). Hosts and vectors may acquire immunity to specific pathogen genome sequences, and may undergo demographic change by natural death and reproduction, as well as migration within complex population structures (Fig. 1D). Pathogens can be transmitted through direct contact among or between hosts and vectors, or through vertical transmission. Pathogens have genomes composed of segments or chromosomes represented by sequences of letters from an arbitrary alphabet, such as DNA bases, amino acids, or other arbitrary alleles. Genomes are capable of mutating, reassorting, and recombining over time. Crucially, individual genome sequences may affect the behavior of their associated pathogens and the hosts and vectors they infect by modifying the rates of any type of event in the simulation, resulting in selection and complex evolutionary dynamics (Fig. 1E). Finally, Opqua contains functions to graph and analyze the evolutionary and epidemiological trajectories of the simulation. The software is presently available as a Python package with documentation, examples, and source code published on GitHub (github.com/pablocarderam/opqua), and a detailed discussion of its functioning in the supplementary materials.

\section{Effect of transmission environment on evolution across fitness valleys}

Using Opqua’s evolutionary modeling capabilities, we examined the epidemiological determinants of pathogen stochastic tunneling across fitness valleys $(37,39)$. In particular, we were interested in understanding whether competition for transmission between pathogens in high transmission environments can inhibit stochastic tunneling. This hypothesis has been proposed as a partial explanation for the seemingly paradoxical way in which antimalarial resistance has emerged in the field. In repeated instances, malaria parasites resistant to different treatments have been first spotted in the low transmission environments of South America, Southeast Asia, and the Pacific, instead of the high-transmission regions in sub-Saharan Africa that contribute the bulk of global malaria cases and treatment $(36,47,48)$. 
A

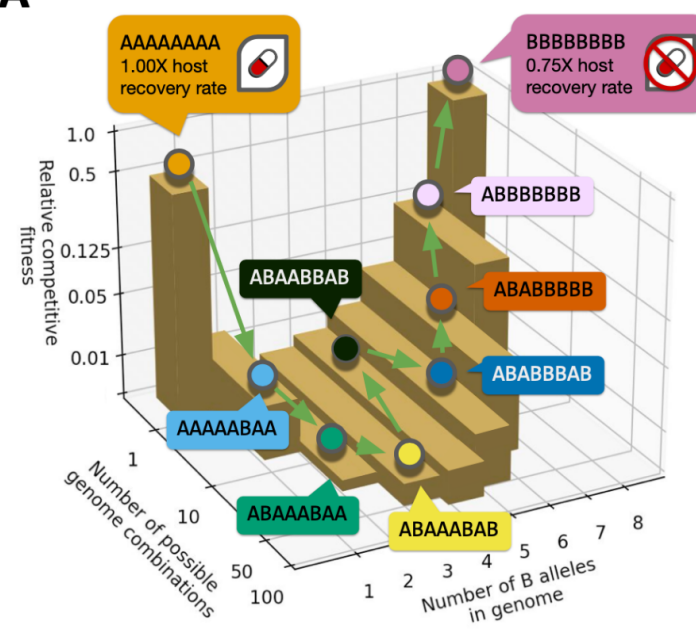

D

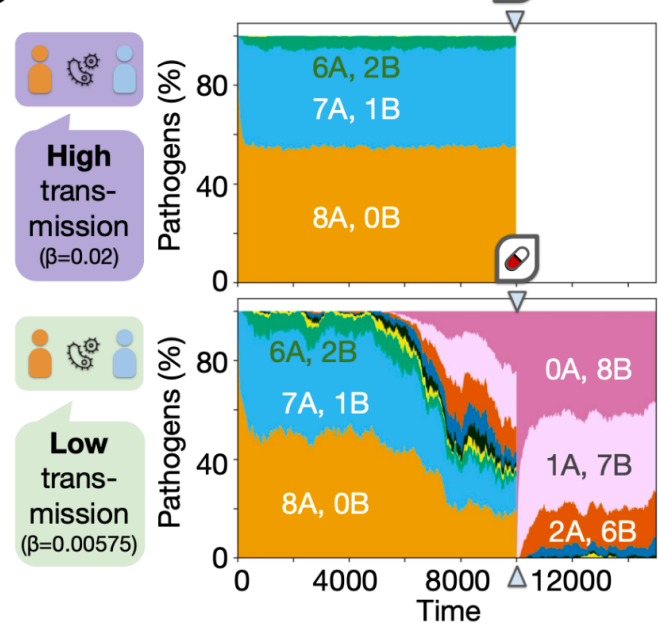

B
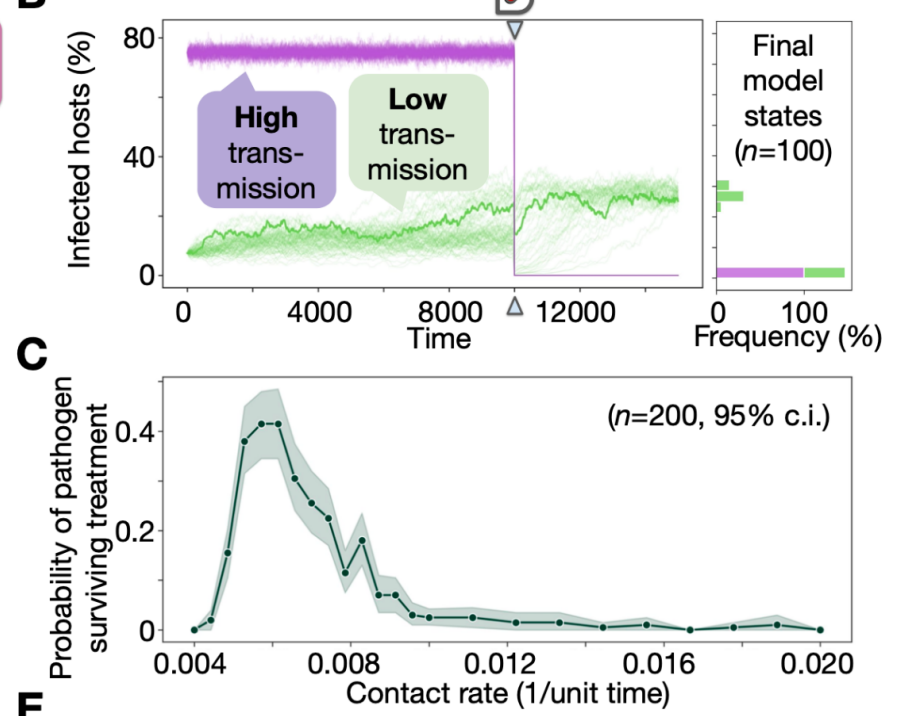

$\mathbf{E}$
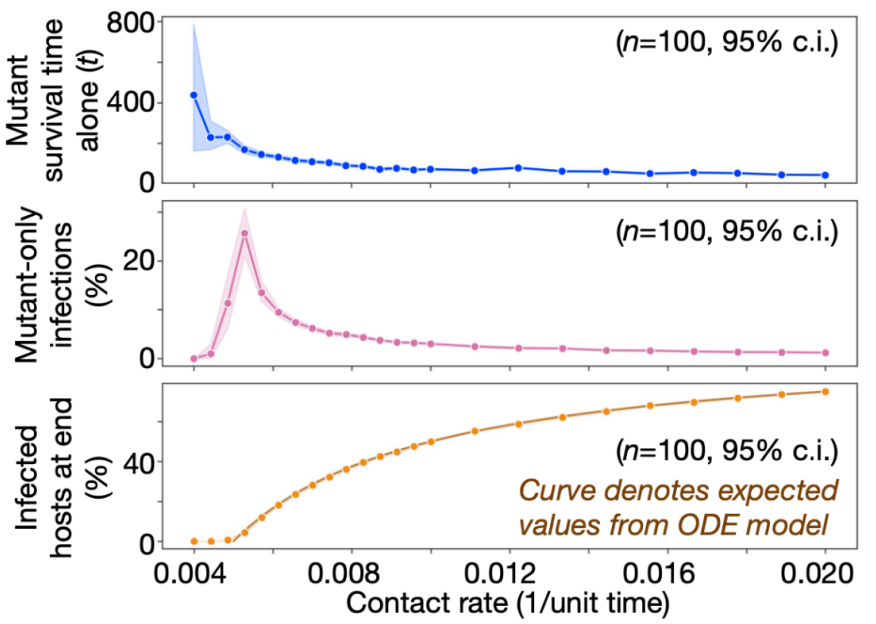

Fig. 2. Competition between pathogens restricts evolution across fitness valleys. (A) A model system contains pathogen genomes with eight different bi-allelic loci. Fitness follows a valley-like distribution according to a genome's " $\mathrm{B}$ " alleles, where the " $0-\mathrm{B}$ " wild-type sequence outcompetes all mutant genomes except for the "8-B" sequence, which encodes for higher competitive fitness, lower recovery rates, and drug resistance. (B) Over time, tracking infections in 1000 hosts shows that pathogens in a high-transmission environment are eradicated during a drug administration event. However, drug effectiveness is halved in a low transmission environment, as resistant mutants can evolve. (C) An optimal, low contact rate favors the evolution of the drug resistance trait in this system. (D) By tracking the genetic composition of the pathogen population over time, we see that at high contact rates $(\beta)$, most mutants remain evolutionarily close to the wild-type, whereas low contact rates lead to the breakthrough and population sweep of distant mutants with high fitness. (E) Even when removing all mutant advantages, the frequency and duration of mutant-only host infections is greater at low contact rates, but is counteracted by low infected host totals. The interplay between these two forces determines stochastic tunneling across the fitness valley. 
With this hypothesis in mind, we established a simple epidemiological system in which pathogens with mutating genomes compete with each other for transmission from host to host as they spread through direct contact within a single population. To model a fitness valley, pathogen genomes in this system consist of eight independent loci, each with two possible alleles (Fig. 2A). Genome fitness follows a valley-like pattern in which an initial fitness peak of "wild-type" genomes is separated by a sequence of mutations from a second, higher fitness peak of "resistant" genomes capable of surviving drug treatment. The intermediate mutations constitute a valley of lower-fitness mutants between the two peaks. When pathogens with different genomes coexist within the same individual, those with more fit genomes have a greater probability of transmission and mutation, due to their greater share of the intra-host pathogen population. Mutant pathogens at the "resistant" peak further increase their fitness through decreased recovery rates. After letting the system evolve for a certain amount of time, we apply a drug treatment that kills all pathogens, save for those with the resistant genome.

By simulating disease outbreaks in this model system, we are able to recapitulate the predicted competitive interference behavior (Figs. 2B, 2C). At high transmission intensities, pathogens fail to evolve resistant genotypes and the infection is cleared from the population after drug treatment. However, low transmission settings allow mutant pathogens to persist and evolve across the fitness valley and reach the more fit, resistant genotype, which is able to survive drug treatment. We can track the genomic composition of populations with different transmission conditions and outcomes over time to observe the evolutionary paths taken in each environment (Fig. 2D). In both high and low transmission environments, we see mutant pathogens are able to evolve and coexist consistently within hosts infected by wild-type pathogens, but are constrained to being one or two mutations away. When transmission rates are high, mutant pathogens are rarely able to survive for long within hosts without wild-type pathogens coinfecting and outcompeting them within the same host. In contrast, low transmission environments allow for mutants to evolve for longer without interference from wild-type pathogens with greater competitive fitness. This leads to a greater frequency of distant mutants in low transmission environments, which eventually result in the appearance and fixation of resistant pathogens. Thus, low transmission environments generate the cryptic genetic variation that has been shown to underlie evolution to new adaptive peaks in other experimental and theoretical models in evolutionary biology $(49,50)$.

However, by varying the contact rate, we can see that the relationship between transmission and evolution is not linear (Fig. 2C). Instead, the likelihood of pathogens evolving resistant genotypes is a result of two opposing forces. On the one hand, there is the likelihood of having hosts infected by low-fitness mutants for long periods of time without wild-type competitors that interfere with further evolution towards more distant genotypes. This likelihood is greater 
in low transmission environments where competition is weak, as long as transmission is strong enough to support a stable pathogen population (Fig. 2E, top and middle). On the other hand, we have the likelihood of survival for strains that reach new fitness peaks. This likelihood is greater in high transmission environments with high numbers of infected hosts, which avoid stochastic extinction events (Fig. 2E, bottom). The balance between these opposed dynamics determines an optimal transmission level for evolution (Fig. 2C), dependent on the features of the adaptive landscape.

In particular, for valley-crossing to be more likely in low transmission regimes, the mutational distance between peaks and the drop in fitness between them must both be large enough to require evolution to occur throughout a transmission chain of multiple hosts. If the distance between peaks is too small or the valley too shallow, pathogens will successfully evolve across the valley within a single host even in the presence of a more fit competitor (Fig. S2). This pattern is exhibited in Fig. 2D with the consistent short-distance evolution at both high and low fitness. Shorter valleys also imply a more restricted number of mutational paths through the valley, which decreases the likelihood of low transmission regimes leading to stochastic tunneling due to their smaller pathogen populations and thus net mutation rates. In this way, high transmission environments are more effective at taking single evolutionary steps with significant fitness differences, whereas low transmission environments are more effective at taking multiple, successive steps with minor differences in fitness. Our results show these small steps are ultimately more effective at tunneling through long evolutionary distances with low overall fitness.

\section{Evolution across descending fitness landscapes in deterministic and stochastic models}

We next focused on the evolutionary dynamics of pathogen populations mutating into low fitness regimes, corresponding to the descent into a fitness valley. We were interested in comparing the capabilities of genomic epidemiological models and traditional epidemiological modeling techniques in the context of stochastic tunneling. To do so, we constructed a compartment model describing a new host-pathogen system with only two possible genotypes: wild-type and mutant. In this system, uninfected hosts can become infected with one or both genotypes (Figs. 3A, S3). Similar to our previous models, mutant pathogens have lower competitive fitness than their wild-type counterparts. 


\section{A}
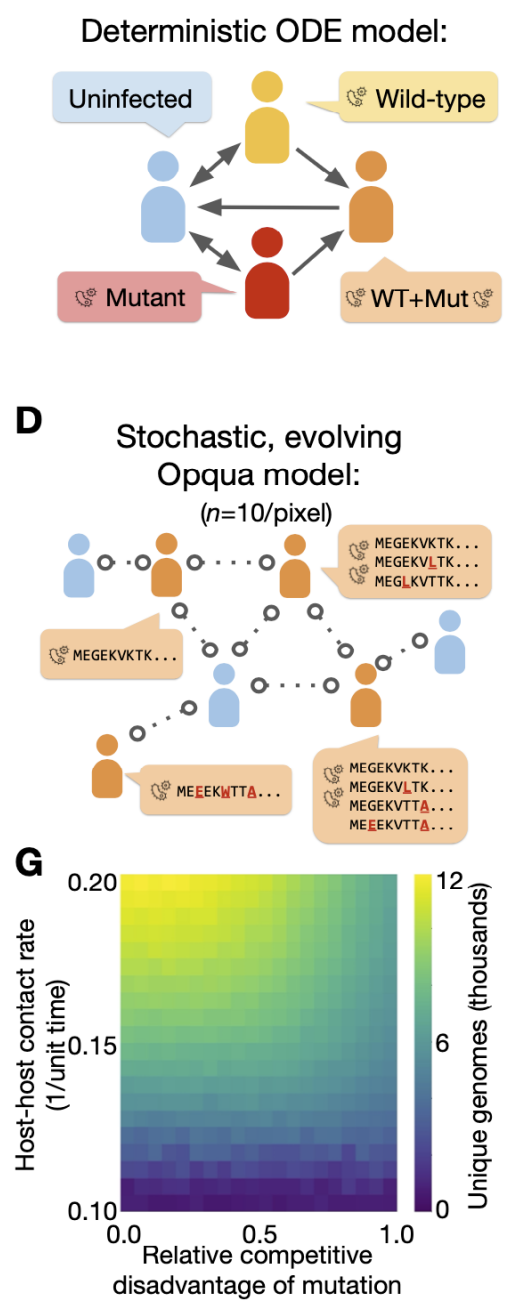
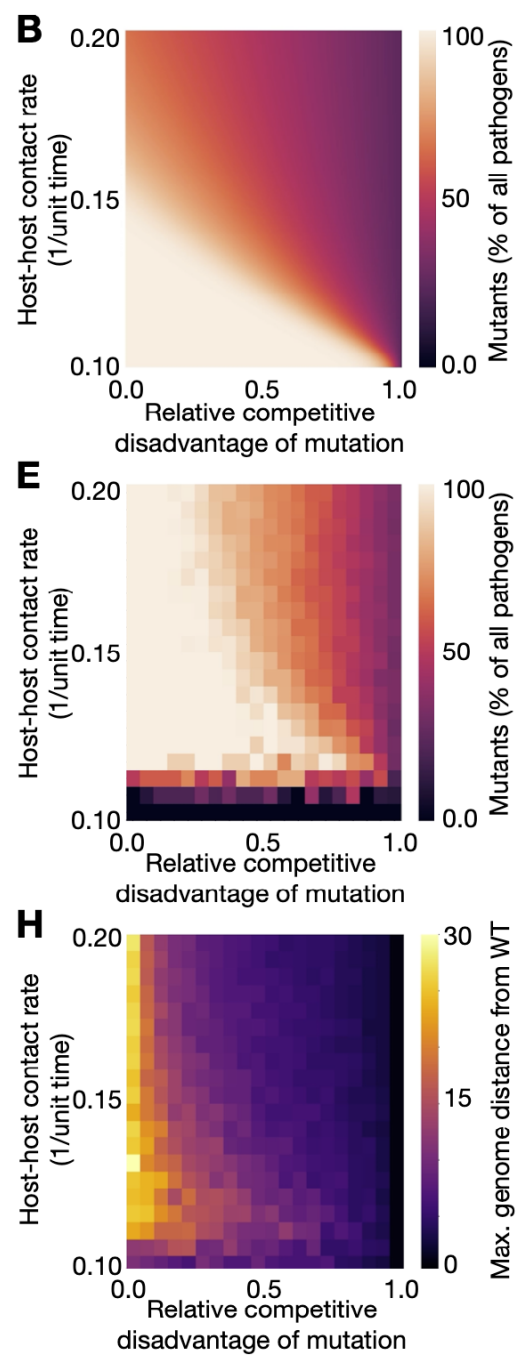
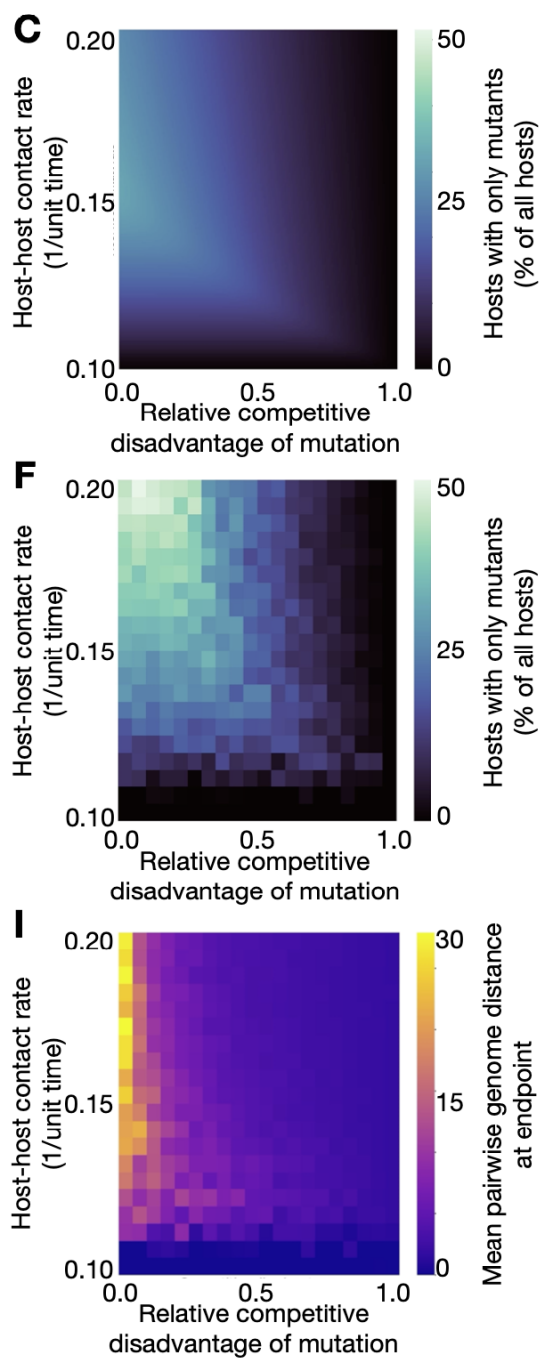

Fig. 3. Deterministic and stochastic simulations describe the effect of competition on the distribution and dimensions of pathogen evolution. (A) Ordinary differential equations (ODE) model the evolution of a pathogen with a single bi-allelic locus. (B) Mutant pathogens are still common at high fitness costs to mutants, as long as contact rates are low. (C) Lone mutants (with no within-host wild-type competitors) are favored at a peak contact rate, which lowers in value as the fitness cost of mutation rises. (D) A stochastic simulation of a similar system tracks evolution across 500 loci, each with 20 possible alleles. Each mutation away from the wild-type sequence reduces fitness by the factor shown. The distributions of mutants among (E) pathogens and (F) hosts show similar behaviors to the ODE model. Stochastic pathogen extinction events drive mutants down at low contact rates, while genetic drift drives them up at high contact rates. Furthermore, simulations allow analysis of the resulting genomes. (G) High contact rates always lead to a higher number of genotypes explored. However, when mutation fitness costs increase, low contact rates allow for the evolution of pathogen lineages more distinct both from $(\mathbf{H})$ the wild-type and (I) each other.

We numerically solved this model as a system of ordinary differential equations (ODEs), varying the relative competitive disadvantage of mutants $(\lambda)$ and the intensity of transmission 
(host-host contact rate $\beta$ ). The results are concordant with our earlier findings, showing that low transmission environments facilitate the survival of unfit mutants in a pathogen population, even when the fitness cost of mutations is considerably high (Fig. 3B). We also examined the fraction of hosts infected with only mutant pathogens, since as we have already seen, this is an important determinant of the ability to tunnel large evolutionary distances at low fitness. In this case, the model shows an optimal transmission intensity at which "mutant-only" infections peak for any given mutation fitness cost (Fig. 3C). Mutants are not as effective at infecting hosts at lower contact rates, while at higher contact rates, wild-type pathogens coinfect and interfere with mutants more often. The optimal transmission level for mutant-only infections decreases as the competitive fitness disadvantage of mutants becomes more prominent, eventually approaching the minimum contact rate required for maintaining stable pathogen populations. This is in line with our earlier results showing higher stochastic tunneling rates at low contact rates in the fitness valley system.

We wanted to ascertain the differences in mutant prevalence at different contact rates arise from competition between wild-type and mutant pathogens instead of other behaviors possibly emerging from a changing contact rate. If our hypothesis were indeed correct, it would follow that varying the fraction of infected hosts (which directly affects the probability of coinfection and competition) while keeping a constant contact rate should achieve similar results. We therefore varied host recovery rates instead of contact rates (Fig. S4). When recovery rates were matched to the corresponding infected host fractions, we observed essentially the same dynamics of mutant prevalence as seen from the contact rates studied. Nevertheless, recovery rates have an additional, smaller effect of their own: at high recovery rates, the effect of increased removal of mutants from the population through recovery becomes visible (Figs. S4C, S4D).

We then constructed stochastic simulations using Opqua to study the same type of behavior. The stochastic simulation was designed to have all epidemiological parameters identical to those in the deterministic ODE model, with the only difference of allowing hosts to become infected with pathogens containing complex genomes simulating a peptide sequence of interest with 500 loci, each with 20 possible amino acid alleles (Fig. 3D). Each mutation decreased pathogen fitness by a set factor, resulting in an exponentially-decreasing fitness landscape away from the wild-type genome sequence.

By plotting the average of multiple simulations at different contact rates and mutant fitness costs, we replicated the behaviors observed in the ODE model using the genomic computational simulations (Fig. 3E, 3F). The results are largely the same, with two differences. First, contact rates that are too low are susceptible to extinction of all pathogen genotypes (counted as a zero 
fraction of mutants) within the simulated timeframe in the stochastic simulations, a phenomenon not observed in the deterministic model. Second, the stochastic simulations show greater mutant prevalence for low mutation fitness costs and higher contact rates than the equivalent parameters in the deterministic model. This is a consequence of genetic drift: if different genomes have comparable fitness and transmission is not a scarce resource, the wild-type genome becomes just one among a large number of comparable, competing genotypes, and is subject to stochastic extinction like any other genotype. This kind of behavior cannot be observed in a deterministic model, nor any model with only two possible genotypes. As a result, genomic epidemiological models like those produced by Opqua are able to more accurately capture the behavior of systems with high genetic dimensionality.

Furthermore, genomic epidemiological simulations allow us to analyze the sequences and distributions of genomes that evolved throughout the simulations. By computing different metrics describing the variation in genomes observed in each simulation, we can construct a quantitative description of the different kinds of evolutionary space explored. For instance, high transmission regimes always result in a higher number of genomes explored, regardless of the fitness cost of mutation (Fig. 3G). This is due to higher numbers of infections providing a greater net mutation frequency. However, even though low contact rates result in fewer mutants, those mutants can evolve further from the wild-type starting point at low contact rates and high fitness costs to mutation (Fig. $3 \mathrm{H}$ ), consistent with our earlier results in the fitness valley model. In addition, the resulting genomes at the end of the simulation are also more different from each other at low contact rates (Fig. 3I). Thus, genomic epidemiological models provide a quantitative description of how transmission environment and within-host competition shape pathogen cryptic variation.

\section{Effects of host population structure and pathogen biology on evolution}

Having established approaches for describing evolution using genomic epidemiological simulations, we considered other kinds of epidemiological determinants of evolution beyond transmission intensity. We first examined two host population characteristics: size and inter-population mobility (Fig. 4). 


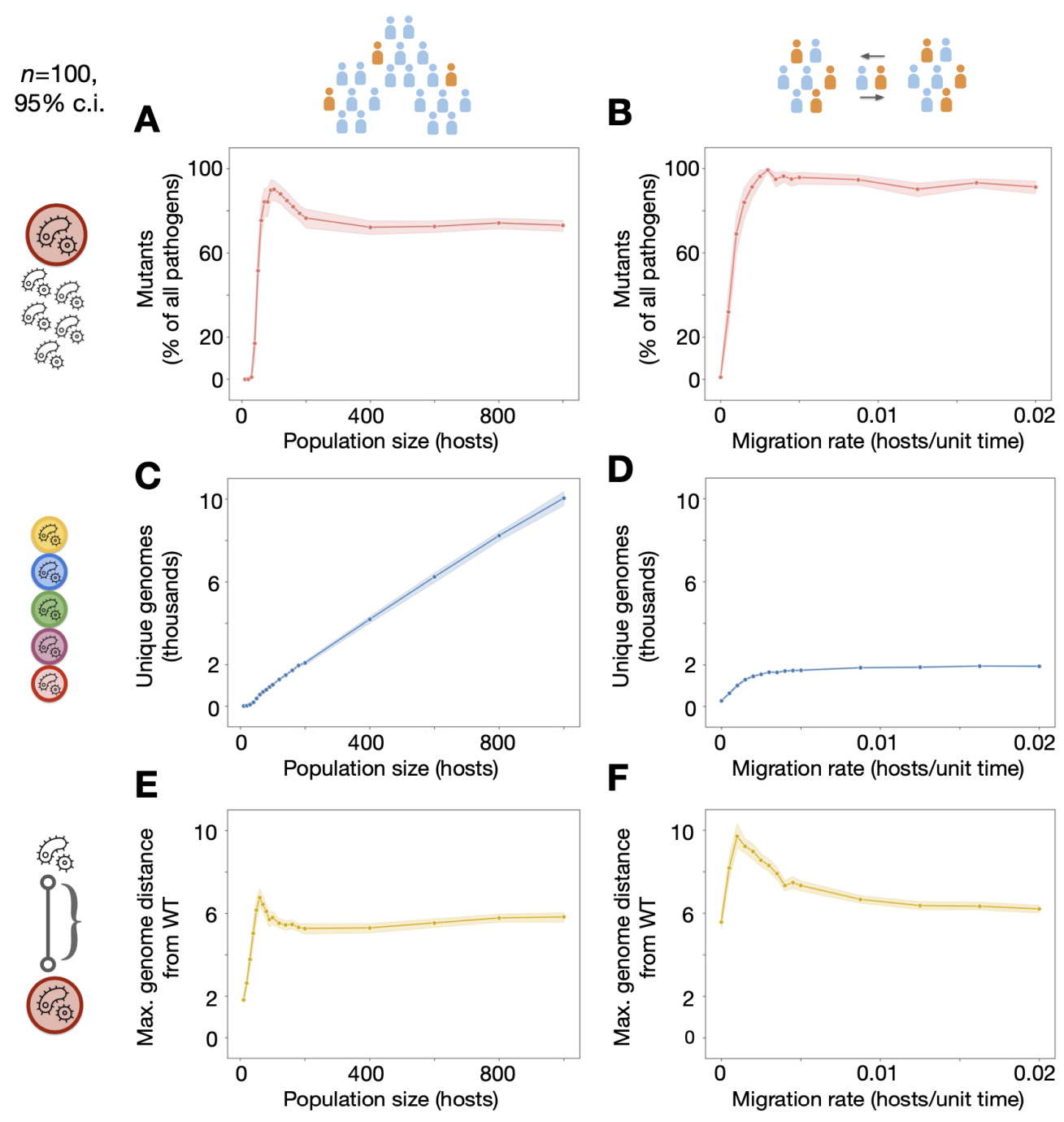

Fig. 4. Population size and structure affect the distribution and dimensions of pathogen evolution. (A) At constant contact rates and relative competitive fitness of mutants, population sizes that are too low cause stochastic extinction of mutants. Mutants increase in frequency as host populations grow, aided by fixation through genetic drift. At larger population sizes, drift leading to wild-type extinction is less likely and the mutant population stabilizes at a lower value. (B) The frequency of mutants within the pathogen population follows a similar pattern when increasing inter-population host migration in a system of 10 interconnected sub-populations, each initially with 10 hosts. (C) An increase in host population size leads to a near-proportional increase in the number of unique pathogen genomes explored. (D) In contrast, increasing mobility leads to the number of genomes explored converging on the value for a single population of 100 hosts. (E) Genome distance from the wild-type sequence follows a similar pattern to mutant fraction when increasing population size. (F) Low migration rates with frequent local extinction and re-colonization events allow pathogens to evolve across longer evolutionary distances than what either the individual population or total metapopulation sizes (10 and 100 hosts, respectively) would normally permit. 
Increasing population sizes initially leads to a greater fraction of mutants among the pathogen population, since small host populations lead to stochastic extinction events for all pathogens (including mutants, counted as a zero fraction of mutants; Fig. 4A). At slightly larger population sizes, the pathogen population is stable enough to avoid complete extinction, but small enough to be susceptible to genetic drift and allow slightly unfit mutants to fixate, driving wild-type pathogens extinct. Lastly, at larger host population sizes the pathogen population is less susceptible to genetic drift and the stochastic extinction of more fit wild-type pathogens. Hence, the fraction of pathogens with mutant genomes decreases slightly to a stable equilibrium. We see similar dynamics by varying host inter-population migration rates in a metapopulation model with a fixed number of total hosts (Fig. 4B). Low mobility rates make the metapopulation system behave like a series of small, isolated populations, while high mobility makes the system behave like a single, well-mixed, large population.

Despite this resemblance in the distribution of mutants in pathogen populations, differences emerge between host population size and mobility when examining genomes. While larger host populations lead to larger infected populations and thus a greater number of unique genomes explored (Fig. 4C), increased mobility does not increase infected hosts, and therefore unique genomes, beyond a certain point (Fig. 4D). The stochastic extinction of high-fitness wild-type pathogens in small populations allows for an early peak in genome distance from wild type, before descending to a slightly lower stable value for larger populations (Fig. 4E). Intriguingly, the most notable increase in the maximum evolutionary distance explored comes from low host mobility systems, provided host mobility is high enough to sustain continued infection (Fig. 4F). Low mobility between host populations results in local pathogen extinctions that allow any mutant pathogens arriving in the population to spread without competition. In this way, the reduced genetic variability initially produced by founder effects paradoxically generates increased variability in the long run. This result is analogous to the effects of low transmission within populations shown in our previous models, with similar evolutionary mechanisms operating on a different scale.

Finally, we used the same approach to examine how different aspects of pathogen biology shape evolution in descending fitness regimes. We focused on the effects of de novo mutation rate, the number of pathogens inoculated during infection, and genomic recombination rates in two modes of transmission: host-host (Fig. S5) and host-vector (Fig. 5). 


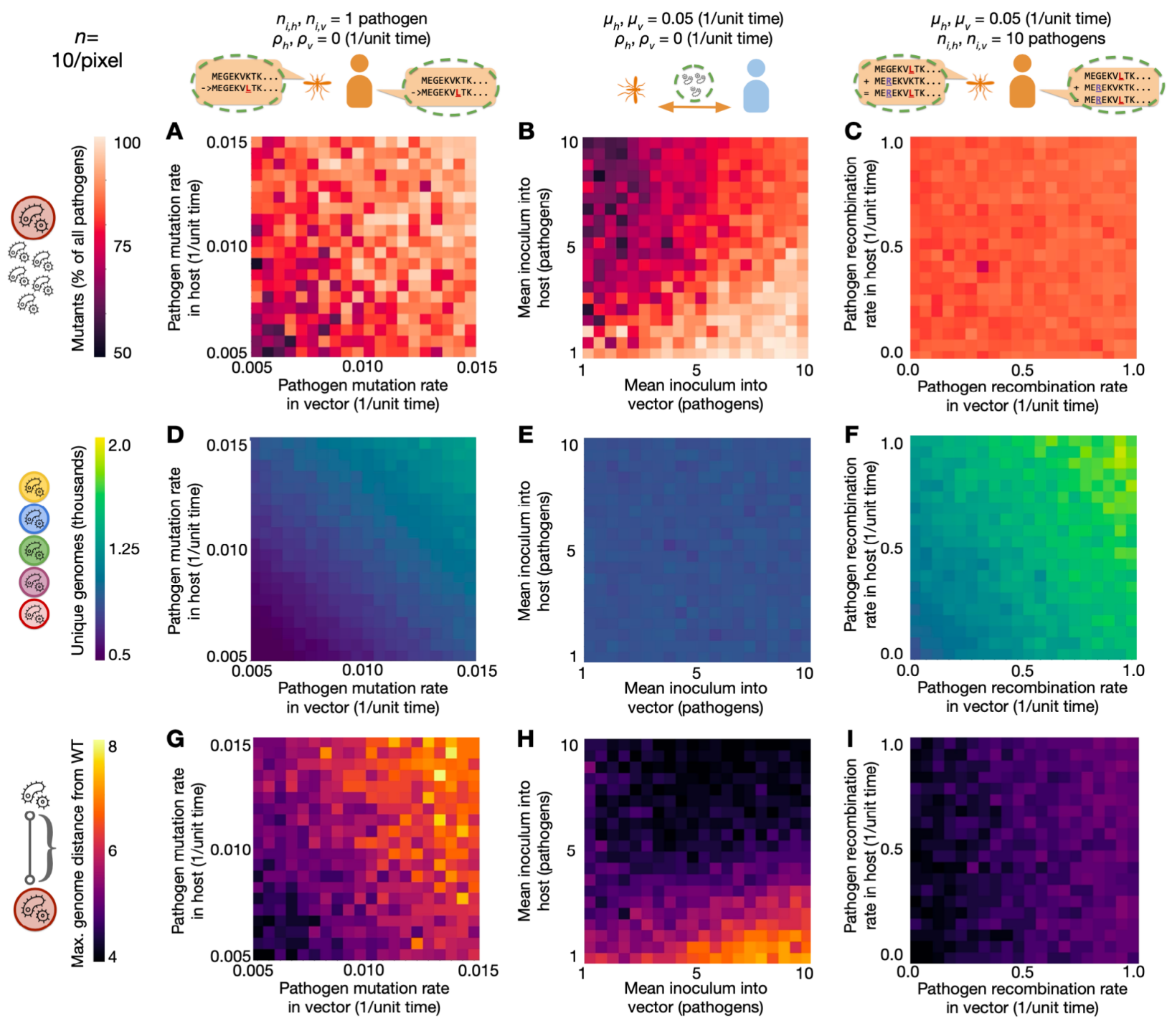

Fig. 5. Pathogen biology and life history affect the distribution and dimensions of evolution. (A) High mutation rates in both hosts $\left(\mu_{h}\right)$ and vectors $\left(\mu_{v}\right)$ lead to greater mutant frequency among pathogens. (B) Greater mean inoculum sizes from hosts (where competitive selection is present) into vectors $\left(n_{v}\right)$ increase mutant frequency by capturing within-host diversity. However, mutant frequency increases at lower inoculum sizes from vectors (where selection is absent) into hosts $\left(n_{h}\right)$, due to bottlenecks reducing intra-host competition. (C) Recombination rates have little effect on mutant frequency. (D) Increasing mutation rates in both hosts and vectors leads to a higher number of genomes explored, while (E) inoculum sizes into both hosts and vectors have no effect. (F) Increased recombination rates in both hosts and vectors lead to an increase in the number of genotypes explored. (G) The maximum evolutionary distance from wild-type (WT) increases with mutation rates. (H) Low inoculums into hosts and high inoculums into vectors are optimal for increasing evolutionary distance, as they combine bottlenecks before selection and capture diversity after it. (I) Increased recombination within vectors (where competitive selection is absent; $\rho_{v}$ ), more so than hosts (where competitive selection is present; $\rho_{h}$ ), leads to higher distances from wild-type. 
We chose to model a host-vector system where competition and selection only occur within hosts, while all genomes have equal competitive fitness within vectors. In this way, we can study the impact of uncoupling selection from these variables in part of the pathogen life cycle. This can be observed in vector-borne diseases, where the vastly different genetic programs of pathogens and host-pathogen interactions across life stages lead to mutations that affect pathogen fitness in hosts but not vectors, or vice-versa.

Unsurprisingly, increasing mutation rates always increase mutant prevalence among pathogens (Figs. 5A, S5A), the number of unique genomes (Figs. 5D, S5D), and the maximum distance from wild-type (Figs. 5G, S5G). Although inoculum sizes show little effect the number of unique mutants explored (Figs. 5E, S5E), the mutant fraction of pathogens and maximum distance show clear patterns. Small inoculum sizes from vectors into hosts are key to increasing mutant fractions of the pathogen population (Figs. 5B, S5B) and maximum evolutionary distance from wild-type (Figs. $5 \mathrm{H}, \mathrm{S} 5 \mathrm{H}$ ). This is due to the fact that small inoculums create a population bottleneck that allows mutants to be transmitted without wild-type competitors. Furthermore, when inoculating from vectors into hosts, pathogens are sampled randomly without a transmission disadvantage for mutants.

Varying inoculum size from hosts into vectors and vice-versa in a host-vector system shows complex interactions between the two variables (Fig. $5 \mathrm{H}$ ). When considering a regime of small inoculums into hosts, a high inoculum into vectors captures greater genetic variation generated in the host, leading to maximized mutant fractions and evolutionary distance from wild-type. Paradoxically, if the mean inoculum into hosts is high, the effect is reversed. In these cases, contacts from infected vectors are more likely to result in coinfections in hosts, leading to competitive exclusion of unfit mutants. Therefore, when inoculums into hosts are high, small inoculums into vectors reestablish the population bottleneck that reduces competition from wild-type pathogens, leading to greater mutant fractions and evolutionary distance from wild-type.

Recombination presents an interesting case in that the dynamics of mutant pathogen fractions and maximum evolutionary distance become uncoupled. Because recombination cannot create de novo genetic variation, mutation rates still largely determine mutant prevalence. This means the effect of recombination on mutant fractions of pathogen populations is minimal (Figs. 5C, S5C, S6A). Nevertheless, recombination rates in both hosts and vectors increase the number of unique genomes explored (Figs. 5F, S5F, S6B). Additionally, recombination can increase the maximum evolutionary distance from wild-type explored by pathogens. In our host-vector system, the effect of recombination on evolutionary distance is largest if recombination occurs within vectors, a life stage where the new genotypes will not be outcompeted by their parents 
(Fig. 5I). Nevertheless, recombination in hosts can also increase the maximum evolutionary distance in host-host models (Fig. S5I). The magnitude of this effect is dependent on the mean inoculum size, as small inoculums remove the within-host variation from which recombination can occur (Fig. S6). This shows recombination can also play a relevant role in stochastic tunneling of infections with the host-host transmission.

\section{Perspectives and outlook}

In this work, we present Opqua, a flexible computational modeling framework capable of simulating genomic epidemiology. We use it to study pathogen competition and evolutionary dynamics in fitness valleys and descending fitness regimes with a quantitative, systematic approach. This allows us to better understand how pathogens acquire new fitness-conferring traits that require multiple separate epistatic mutations, a process known as stochastic tunneling $(37,39)$. We find that competition between pathogens in settings with high disease prevalence can pose a significant barrier to evolution in certain adaptive landscapes, as suggested by other studies (36). We establish the potential of genomic epidemiological models to provide more complete descriptions of simulated evolution than traditional epidemiological compartment modeling. We then use these descriptions to examine how different aspects of host populations and pathogen biology can alter the competitive and genetic population dynamics that shape evolution. We show that stochastic effects in small, relatively isolated, structured populations can increase the evolutionary distance traversed by pathogens, exploring evolutionary space in greater depth. Lastly, we show how decoupling selection from transmission in pathogen life cycles with multiple stages changes the evolutionary dynamics across stretches of low fitness.

These findings suggest distinct strategies with evolutionary advantages for different kinds of pathogens. For pathogens that depend on direct contact between hosts, such as respiratory viral infections, inoculum sizes are often inextricably tied to transmissibility, resulting in a trade-off between transmission and stochastic tunneling to new adaptive peaks. Conversely, infections with complex life cycles can randomly subsample genetic variability within life stages where selective pressure is weak to increase the maximum evolutionary distance explored, leading to more stochastic tunneling. This can be seen in malaria parasites, which undergo strong population bottlenecks in mosquitoes and within human livers, before intra-host competition in the bloodstream occurs. These bottlenecks reduce the number of individual pathogens by ten orders of magnitude from its highest point within the human bloodstream (51). The findings indicate that interventions that target these vector stages and 
their bottlenecks not only reduce disease prevalence, but can severely hamper pathogen evolvability if they introduce selective pressures on traits that affect host stages.

Our results have important eco-epidemiological implications when considering the effect of transmission conditions, population structure, and mobility in the evolution of infectious diseases. When considering the evolution of distant pathogen genotypes with higher fitness through stochastic tunneling, rural communities consisting of small, semi-isolated groups with relatively low transmission may be more at risk than larger populations with high transmission. Although the recent emergence of SARS-CoV-2 variant of concern Omicron was doubtlessly facilitated by other variables, most notably acquired immunity (52), it is interesting to consider that it may well be an example of this kind of process. Regardless of whether it is, our results suggest that novel, evolutionary distant variants could be likely to emerge in the places where detection would be most challenging: fragmented, mobile populations in regions with relatively low incidence and cryptic transmission of low-fitness genotypes. It is, therefore, perhaps unsurprising that the chain of mutations that led to Omicron went undetected, as the last common ancestor between Omicron and other sequenced variants is likely more than one year old [nextstrain.org/groups/neherlab/ncov/21K.Omicron, $(22,53)$ ]. In this light, monitoring these kinds of peripheral communities could be of even greater value for genomic surveillance programs than monitoring large, central hubs.

A final corollary of these results is that under certain conditions, pathogen evolvability increases the more elimination efforts progress against it. The low-transmission environments brought about by eradication campaigns are the exact conditions needed for stochastic tunneling. This does not in any way imply that eradication efforts are pointless-after all, evolution is always a possibility, while disease burden is a certainty. Nevertheless, despite initial successes, it might help explain why elimination campaigns are so challenging to complete.

We deliberately chose to focus on intra-host competition, as this is the minimum requirement for blocking stochastic tunneling through competitive exclusion: without it, parallel outbreaks for all pathogen genotypes would proceed undeterred in a system with no acquired immunity. Therefore, we avoided using Opqua's ability to modify the intrinsic transmissibility of pathogens according to genome sequence, even though this would increase the magnitude of the effects studied. In addition, by choosing to center on competitive dynamics between pathogens, we opted to keep the effects of host acquired immunity beyond the scope of this study, even though Opqua has the capabilities to simulate them. However, acquired immune selection clearly plays a central role in the evolution of these systems by shifting the fitness landscape over time. This has been proposed in the context of the evolution of malaria parasites 
(36) and is doubtless a crucial factor in the emergence of SARS-CoV-2 variants that evade acquired immune responses (52). Finally, we chose to explore idealized disease models rather than fit a specific disease both for generalizability and computational feasibility. Future work improving the computational performance of these kinds of modeling approaches may allow for models that more closely fit real-world epidemiological and genetic data. Nevertheless, we hope this work helps establish the potential of genomic epidemiological models as a tool to test scenarios, explore hypotheses, and understand the relationship between pathogen evolution and epidemiology.

\section{References:}

1. J. Quick, N. J. Loman, S. Duraffour, J. T. Simpson, E. Severi, L. Cowley, J. A. Bore, R. Koundouno, G. Dudas, A. Mikhail, others, Real-time, portable genome sequencing for Ebola surveillance. Nature. 530, 228-232 (2016).

2. B. Diallo, D. Sissoko, N. J. Loman, H. A. Bah, H. Bah, M. C. Worrell, lya S. Conde, R. Sacko, S. Mesfin, A. Loua, J. K. Kalonda, N. A. Erondu, B. A. Dahl, S. Handrick, I. Goodfellow, L. W. Meredith, M. Cotten, U. Jah, R. E. Guetiya Wadoum, P. Rollin, N. Magassouba, D. Malvy, X. Anglaret, M. W. Carroll, R. B. Aylward, M. H. Djingarey, A. Diarra, P. Formenty, S. Keïta, S. Günther, A. Rambaut, S. Duraffour, Resurgence of Ebola Virus Disease in Guinea Linked to a Survivor With Virus Persistence in Seminal Fluid for More Than 500 Days. Clin. Infect. Dis. 63, 1353-1356 (2016).

3. A. Keita, A. Düx, H. Diallo, S. Calvignac-Spencer, M. Sow, M. Keita, Y. Sidibe, A. Ayouba, F. Lemarcis, A. Marí Saéz, others, Resurgence of Ebola virus in guinea after 5 years calls for careful attention to survivors without creating further stigmatization. Virological (2021).

4. L. Henden, S. Lee, I. Mueller, A. Barry, M. Bahlo, Identity-by-descent analyses for measuring population dynamics and selection in recombining pathogens. PLoS Genet. 14, e1007279 (2018).

5. A. R. Taylor, S. F. Schaffner, G. C. Cerqueira, S. C. Nkhoma, T. J. Anderson, K. Sriprawat, A. Pyae Phyo, F. Nosten, D. E. Neafsey, C. O. Buckee, Quantifying connectivity between local Plasmodium falciparum malaria parasite populations using identity by descent. PLoS Genet. 13, e1007065 (2017).

6. A. R. Taylor, D. F. Echeverry, T. J. C. Anderson, D. E. Neafsey, C. O. Buckee, Identity-by-descent relatedness estimates with uncertainty characterise departure from isolation-by-distance between Plasmodium falciparum populations on the Colombian-Pacific coast. bioRxiv (2020), doi:10.1101/2020.04.10.035303.

7. E. Ghedin, N. A. Sengamalay, M. Shumway, J. Zaborsky, T. Feldblyum, V. Subbu, D. J. Spiro, J. Sitz, H. Koo, P. Bolotov, others, Large-scale sequencing of human influenza reveals the 
dynamic nature of viral genome evolution. Nature. 437, 1162-1166 (2005).

8. R. A. Neher, T. Bedford, Nextflu: real-time tracking of seasonal influenza virus evolution in humans. Bioinformatics. 31, 3546-3548 (2015).

9. Á. O’Toole, V. Hill, O. G. Pybus, A. Watts, I. I. Bogoch, K. Khan, J. P. Messina, The COVID-19 Genomics UK (COG-UK) consortium, Network for Genomic Surveillance in South Africa (NGS-SA), Brazil-UK CADDE Genomic Network, H. Tegally, R. R. Lessells, J. Giandhari, S. Pillay, K. A. Tumedi, G. Nyepetsi, M. Kebabonye, M. Matsheka, M. Mine, S. Tokajian, H. Hassan, T. Salloum, G. Merhi, J. Koweyes, J. L. Geoghegan, J. de Ligt, X. Ren, M. Storey, N. E. Freed, C. Pattabiraman, P. Prasad, A. S. Desai, R. Vasanthapuram, T. F. Schulz, L. Steinbrück, T. Stadler, Swiss Viollier Sequencing Consortium, A. Parisi, A. Bianco, D. García de Viedma, S. Buenestado-Serrano, V. Borges, J. Isidro, S. Duarte, J. P. Gomes, N. S. Zuckerman, M. Mandelboim, O. Mor, T. Seemann, A. Arnott, J. Draper, M. Gall, W. Rawlinson, I. Deveson, S. Schlebusch, J. McMahon, L. Leong, C. K. Lim, M. Chironna, D. Loconsole, A. Bal, L. Josset, E. Holmes, K. St. George, E. Lasek-Nesselquist, R. S. Sikkema, B. Oude Munnink, M. Koopmans, M. Brytting, V. Sudha rani, S. Pavani, T. Smura, A. Heim, S. Kurkela, M. Umair, M. Salman, B. Bartolini, M. Rueca, C. Drosten, T. Wolff, O. Silander, D. Eggink, C. Reusken, H. Vennema, A. Park, C. Carrington, N. Sahadeo, M. Carr, G. Gonzalez, SEARCH Alliance San Diego, National Virus Reference Laboratory, SeqCOVID-Spain, Danish Covid-19 Genome Consortium (DCGC), Communicable Diseases Genomic Network (CDGN), Dutch National SARS-CoV-2 surveillance program, Division of Emerging Infectious Diseases (KDCA), T. de Oliveira, N. Faria, A. Rambaut, M. U. G. Kraemer, Tracking the international spread of SARS-CoV-2 lineages B.1.1.7 and B.1.351/501Y-V2 with grinch. Wellcome Open Res. 6, 121 (2021).

10. M. U. Kraemer, V. Hill, C. Ruis, S. Dellicour, S. Bajaj, J. T. McCrone, G. Baele, K. V. Parag, A. L. Battle, B. Gutierrez, others, Spatiotemporal invasion dynamics of SARS-CoV-2 lineage B. 1.1. 7 emergence. Science (2021), doi:10.1126/science.abj0113.

11. H. Tegally, E. Wilkinson, R. J. Lessells, J. Giandhari, S. Pillay, N. Msomi, K. Mlisana, J. N. Bhiman, A. von Gottberg, S. Walaza, others, Sixteen novel lineages of SARS-CoV-2 in South Africa. Nat. Med. 27, 440-446 (2021).

12. R. J. Rockett, A. Arnott, C. Lam, R. Sadsad, V. Timms, K.-A. Gray, J.-S. Eden, S. Chang, M. Gall, J. Draper, others, Revealing COVID-19 transmission in Australia by SARS-CoV-2 genome sequencing and agent-based modeling. Nat. Med. 26, 1398-1404 (2020).

13. N. F. Müller, C. Wagner, C. D. Frazar, P. Roychoudhury, J. Lee, L. H. Moncla, B. Pelle, M. Richardson, E. Ryke, H. Xie, others, Viral genomes reveal patterns of the SARS-CoV-2 outbreak in Washington State. Sci. Transl. Med. 13 (2021), doi:10.1126/scitranslmed.abf0202.

14. J. E. Lemieux, K. J. Siddle, B. M. Shaw, C. Loreth, S. F. Schaffner, A. Gladden-Young, G. Adams, T. Fink, C. H. Tomkins-Tinch, L. A. Krasilnikova, K. C. DeRuff, M. Rudy, M. R. 
Bauer, K. A. Lagerborg, E. Normandin, S. B. Chapman, S. K. Reilly, M. N. Anahtar, A. E. Lin, A. Carter, C. Myhrvold, M. E. Kemball, S. Chaluvadi, C. Cusick, K. Flowers, A. Neumann, F. Cerrato, M. Farhat, D. Slater, J. B. Harris, J. A. Branda, D. Hooper, J. M. Gaeta, T. P. Baggett, J. O’Connell, A. Gnirke, T. D. Lieberman, A. Philippakis, M. Burns, C. M. Brown, J. Luban, E. T. Ryan, S. E. Turbett, R. C. LaRocque, W. P. Hanage, G. R. Gallagher, L. C. Madoff, S. Smole, V. M. Pierce, E. Rosenberg, P. C. Sabeti, D. J. Park, B. L. MacInnis, Phylogenetic analysis of SARS-CoV-2 in Boston highlights the impact of superspreading events. Science. 371, eabe3261 (2021).

15. J. D. Ramírez, C. Florez, M. Muñoz, C. Hernández, A. Castillo, S. Gomez, A. Rico, L. Pardo, E. C. Barros, S. Castañeda, others, The arrival and spread of SARS-CoV-2 in Colombia. J. Med. Virol. 93, 1158-1163 (2021).

16. J.-M. Kim, S. Y. Park, D. Lee, J.-S. Kim, Y. Park, J. Gwack, M. Y. Kim, D. H. Song, S. T. Jeong, Y.-S. Chung, C. K. Yoo, H. Y. Lee, M.-G. Han, Genomic investigation of the coronavirus disease-2019 outbreak in the Republic of Korea. Sci. Rep. 11, 6009 (2021).

17. S. Kumar, Q. Tao, S. Weaver, M. Sanderford, M. A. Caraballo-Ortiz, S. Sharma, S. L. K. Pond, S. Miura, An Evolutionary Portrait of the Progenitor SARS-CoV-2 and Its Dominant Offshoots in COVID-19 Pandemic. Mol. Biol. Evol. (2021), doi:10.1093/molbev/msab118.

18. L. Pipes, H. Wang, J. P. Huelsenbeck, R. Nielsen, Assessing uncertainty in the rooting of the SARS-CoV-2 phylogeny. Mol. Biol. Evol. 38, 1537-1543 (2021).

19. B. Morel, P. Barbera, L. Czech, B. Bettisworth, L. Hübner, S. Lutteropp, D. Serdari, E.-G. Kostaki, I. Mamais, A. M. Kozlov, others, Phylogenetic analysis of SARS-CoV-2 data is difficult. Mol. Biol. Evol. 38, 1777-1791 (2021).

20. Y. Shu, J. McCauley, GISAID: Global initiative on sharing all influenza data-from vision to reality. Eurosurveillance. 22, 30494 (2017).

21. S. F. Schaffner, A. R. Taylor, W. Wong, D. F. Wirth, D. E. Neafsey, hmmIBD: software to infer pairwise identity by descent between haploid genotypes. Malar. J. 17, 1-4 (2018).

22. J. Hadfield, C. Megill, S. M. Bell, J. Huddleston, B. Potter, C. Callender, P. Sagulenko, T. Bedford, R. A. Neher, Nextstrain: real-time tracking of pathogen evolution. Bioinformatics. 34, 4121-4123 (2018).

23. Á. O’Toole, E. Scher, A. Underwood, B. Jackson, V. Hill, J. T. McCrone, R. Colquhoun, C. Ruis, K. Abu-Dahab, B. Taylor, others, Assignment of Epidemiological Lineages in an Emerging Pandemic Using the Pangolin Tool. Virus Evol. (2021), doi:10.1093/ve/veab064.

24. B. Espinoza, C. Castillo-Chavez, C. Perrings, Mobility restrictions for the control of epidemics: when do they work? Plos One. 15, e0235731 (2020).

25. D. H. Morris, F. W. Rossine, J. B. Plotkin, S. A. Levin, Optimal, near-optimal, and robust epidemic control. Commun. Phys. 4, 78 (2021).

26. C. E. Wagner, C. M. Saad-Roy, S. E. Morris, R. E. Baker, M. J. Mina, J. Farrar, E. C. Holmes, O. G. Pybus, A. L. Graham, E. J. Emanuel, S. A. Levin, C. J. E. Metcalf, B. T. Grenfell, 
Vaccine nationalism and the dynamics and control of SARS-CoV-2. Science. 373, eabj7364 (2021).

27. J. Lourenço, P. S. Wikramaratna, S. Gupta, MANTIS: an R package that simulates multilocus models of pathogen evolution. BMC Bioinformatics. 16, 1-7 (2015).

28. W. Wong, E. A. Wenger, D. L. Hartl, D. F. Wirth, Modeling the genetic relatedness of Plasmodium falciparum parasites following meiotic recombination and cotransmission. PLoS Comput. Biol. 14, e1005923 (2018).

29. A. Jariani, C. Warth, K. Deforche, P. Libin, A. J. Drummond, A. Rambaut, F. A. Matsen IV, K. Theys, SANTA-SIM: simulating viral sequence evolution dynamics under selection and recombination. Virus Evol. 5, vez003 (2019).

30. R. Verity, S. Berube, D. Larremore, SIMPLEGEN (mrc-ide.github.io/SIMPLEGEN/index.html).

31. C. J. Worby, T. D. Read, 'SEEDY'(Simulation of Evolutionary and Epidemiological Dynamics): An R Package to Follow Accumulation of Within-Host Mutation in Pathogens. PloS One. 10, e0129745 (2015).

32. G. Danesh, E. Saulnier, O. Gascuel, M. Choisy, S. Alizon, “Simulating trajectories and phylogenies from population dynamics models with TiPS” (preprint, Bioinformatics, 2020), , doi:10.1101/2020.11.09.373795.

33. B. Peng, M. Kimmel, simuPOP: a forward-time population genetics simulation environment. Bioinformatics. 21, 3686-3687 (2005).

34. B. Peng, C. I. Amos, Forward-time simulations of non-random mating populations using simuPOP. Bioinformatics. 24, 1408-1409 (2008).

35. F. Zanini, R. A. Neher, FFPopSim: an efficient forward simulation package for the evolution of large populations. Bioinformatics. 28, 3332-3333 (2012).

36. A. O. B. Whitlock, J. J. Juliano, N. Mideo, Immune selection suppresses the emergence of drug resistance in malaria parasites but facilitates its spread. PLOS Comput. Biol. 17, e1008577 (2021).

37. Y. Iwasa, F. Michor, M. A. Nowak, Stochastic Tunnels in Evolutionary Dynamics. Genetics. 166, 1571-1579 (2004).

38. C. S. Gokhale, Y. Iwasa, M. A. Nowak, A. Traulsen, The pace of evolution across fitness valleys. J. Theor. Biol. 259, 613-620 (2009).

39. Y. Guo, M. Vucelja, A. Amir, Stochastic tunneling across fitness valleys can give rise to a logarithmic long-term fitness trajectory. Sci. Adv. 5, eaav3842 (2019).

40. B. Steinberg, M. Ostermeier, Environmental changes bridge evolutionary valleys. Sci. Adv. 2, e1500921 (2016).

41. R. Burioni, E. J. Topol, Has SARS-CoV-2 reached peak fitness? Nat. Med. 27, 1323-1324 (2021).

42. J. Zahradník, S. Marciano, M. Shemesh, E. Zoler, D. Harari, J. Chiaravalli, B. Meyer, Y. 
Rudich, C. Li, I. Marton, O. Dym, N. Elad, M. G. Lewis, H. Andersen, M. Gagne, R. A. Seder, D. C. Douek, G. Schreiber, SARS-CoV-2 variant prediction and antiviral drug design are enabled by RBD in vitro evolution. Nat. Microbiol. 6, 1188-1198 (2021).

43. D. P. Martin, S. Lytras, A. G. Lucaci, W. Maier, B. Grüning, S. D. Shank, S. Weaver, O. A. MacLean, R. J. Orton, P. Lemey, M. F. Boni, H. Tegally, G. Harkins, C. Scheepers, J. N. Bhiman, J. Everatt, D. G. Amoako, J. E. San, J. Giandhari, A. Sigal, NGS-SA, C. Williamson, N. Hsiao, A. von Gottberg, A. De Klerk, R. W. Shafer, D. L. Robertson, R. J. Wilkinson, B. T. Sewell, R. Lessells, A. Nekrutenko, A. Greaney, T. Starr, J. Bloom, B. Murrell, E. Wilkinson, T. de Oliveira, S. L. Kosakovsky Pond, Selection analysis identifies significant mutational changes in Omicron that are likely to influence both antibody neutralization and Spike function (part 1 of 2). Virological.org (2021), (available at https://virological.org/t/selection-analysis-identifies-significant-mutational-changes-in-o micron-that-are-likely-to-influence-both-antibody-neutralization-and-spike-function-pa rt-1-of-2/771).

44. A. K. Alame Emane, X. Guo, H. E. Takiff, S. Liu, Drug resistance, fitness and compensatory mutations in Mycobacterium tuberculosis. Tuberculosis. 129, 102091 (2021).

45. P. Mesén-Ramírez, B. Bergmann, M. Elhabiri, L. Zhu, H. von Thien, C. Castro-Peña, T.-W. Gilberger, E. Davioud-Charvet, Z. Bozdech, A. Bachmann, T. Spielmann, The parasitophorous vacuole nutrient channel is critical for drug access in malaria parasites and modulates the artemisinin resistance fitness cost. Cell Host Microbe. 29, 1774-1787.e9 (2021).

46. D. F. Gómez Aldana, Opqua - Diccionario muysca - español. Dicc. Muysca - Esp., (available at http://muysca.cubun.org/opqua).

47. T. J. C. Anderson, C. Roper, The origins and spread of antimalarial drug resistance: Lessons for policy makers. Acta Trop. 94, 269-280 (2005).

48. E. A. Ashley, M. Dhorda, R. M. Fairhurst, C. Amaratunga, P. Lim, S. Suon, S. Sreng, J. M. Anderson, S. Mao, B. Sam, others, Spread of artemisinin resistance in Plasmodium falciparum malaria. N. Engl. J. Med. 371, 411-423 (2014).

49. J. Zheng, J. L. Payne, A. Wagner, Cryptic genetic variation accelerates evolution by opening access to diverse adaptive peaks. Science. 365, 347-353 (2019).

50. A. B. Paaby, M. V. Rockman, Cryptic genetic variation: evolution's hidden substrate. Nat. Rev. Genet. 15, 247-258 (2014).

51. W. Graumans, E. Jacobs, T. Bousema, P. Sinnis, When Is a Plasmodium-Infected Mosquito an Infectious Mosquito? Trends Parasitol. 36, 705-716 (2020).

52. S. Cele, F. Karim, G. Lustig, J. E. San, T. Hermanus, H. Tegally, J. Snyman, T. Moyo-Gwete, E. Wilkinson, M. Bernstein, K. Khan, S.-H. Hwa, S. W. Tilles, L. Singh, J. Giandhari, N. Mthabela, M. Mazibuko, Y. Ganga, B. I. Gosnell, S. A. Karim, W. Hanekom, W. C. Van Voorhis, T. Ndung'u, COMMIT-KZN Team, R. J. Lessells, P. L. Moore, M.-Y. S. Moosa, T. de 
Oliveira, A. Sigal, medRxiv, in press, doi:10.1101/2021.09.14.21263564.

53. E. B. Hodcroft, CoVariants: SARS-CoV-2 Mutations and Variants of Interest (2021; https://covariants.org/).

\section{Acknowledgements:}

The authors would like to gratefully acknowledge Manuela Carrasquilla, Vladimir Corredor, Juan Estupiñán, Alejandro Feged Rivadeneira, Felipe González Casabianca, Angélica Knudson, and David Suárez for their valuable insights throughout the development of this project.

\section{Funding:}

P.C. is supported by the Surpina and Panos Eurnekian Biotechnology Fund Fellowship at the Massachusetts Institute of Technology. MSV is supported by the International Development Research Centre (Centre de recherches pour le développement international) - Grant \#109582-001

\section{Authors contributions:}

P.C. and M.S.V. conceived the idea of the software, designed the study, and wrote the manuscript. P.C. implemented the code.

\section{Competing interests:}

None declared.

\section{Data and materials availability:}

All code necessary to install and use the Opqua modeling framework is available at https://github.com/pablocarderam/opqua. All data and code necessary to reproduce the simulations and analyses of this specific study is available at https://github.com/pablocarderam/fitness valleys opqua/.

\section{List of Supplementary materials:}

Materials and Methods

Fig S1 - S3

Tables S1 - S3

References (26-32) 


\section{Supplementary Materials:}

\section{Materials and Methods}

To address the questions posed in this study, we developed Opqua, an epidemiological modeling framework for pathogen population genetics and evolution. Opqua stochastically simulates pathogens with distinct, evolving genotypes that spread through host populations with specific acquired immune profiles. Opqua is available for download through PyPI (pypi.org/project/opqua) with installation, usage instructions, and source code published on GitHub (github.com/pablocarderam/opqua).

\section{Basic model concepts}

Opqua models are composed of populations containing hosts and/or vectors, which themselves may be infected by a number of pathogens with different genomes.

A genome is represented as a string of characters. All genomes must be of the same length (a set number of loci), and each position within the genome can have one of many different characters specified by the user (corresponding to different alleles). Different loci in the genome may have different possible alleles available to them. Genomes may be composed of separate chromosomes or genome segments separated by the "/" character, which is reserved for this purpose.

Each population may have its own unique parameters dictating the events that happen inside of it, including how pathogens are spread between its hosts and vectors.

\section{Model events}

There are different kinds of events that may occur to hosts and vectors in a population:

- contact between an infectious host/vector and another host/vector in the same population (intra-population contact, or "contact") or in a different population (inter-population contact, or "population contact")

- migration of a host/vector from one population to another

- recovery of an infected host/vector

- birth of a new host/vector from an existing host/vector

- death of a host/vector due to pathogen infection or by "natural" causes

- mutation of a pathogen in an infected host/vector

- recombination of two pathogens in an infected host/vector 
The likelihood of each event occurring is determined by the population's parameters (explained in the online documentation) and the number of infected and healthy hosts and/or vectors in the population(s) involved. Crucially, it is also determined by the genome sequences of the pathogens infecting those hosts and vectors. The user may specify arbitrary functions to evaluate how a genome sequence affects any of the above kinds of rates. As an example, a specific genome sequence may result in increased transmission within populations but decreased migration of infected hosts, or increased mutation rates. These custom functions may be different across populations, resulting in different adaptive landscapes within different populations.

Contacts within and between populations may happen by any combination of host-host, host-vector, and/or vector-host routes, depending on the populations' parameters. When a contact occurs, each pathogen genome present in the infecting host/vector is transferred to the receiving host/vector as long as one "infectious unit" is inoculated. The number of infectious units inoculated is randomly distributed based on a Poisson probability distribution. The mean of this distribution is set by the receiving host/vector's population parameters. The genomes chosen for transmission are sampled randomly according to the fraction of intra-host fitness each genome contributes to the total within the host or vector.

Inter-population contacts occur via the same mechanism as intra-population contacts, with the distinction that the two populations must be linked in a compatible way. For example, if a vector-borne model with two separate populations allows vectors from Population A to contact hosts in Population B, then the vector-host population contact rate in Population A and the host-vector population contact rate in Population B must both be greater than zero. Migration of hosts/vectors from one population to another depends on a single rate defining the frequency of vector/host transport events from a given population to another. Therefore, Population A would have a specific migration rate dictating transport to Population $\mathrm{B}$, and Population B would have a separate rate governing transport towards A.

The recovery of an infected host or vector results in all pathogens being removed from the host/vector. Additionally, the host/vector may optionally gain protection from pathogens that contain specific genome sequences present in the genomes of the pathogens it recovered from, representing immune memory. The user may specify a population parameter delimiting the contiguous loci in the genome that are saved on the recovered host/vector as "protection sequences". Pathogens containing any of the host/vector's protection sequences will not be able to infect the host/vector. 
Births result in a new host/vector that may optionally inherit its parent's protection sequences. Additionally, a parent may optionally infect its offspring at birth following a Poisson sampling process equivalent to the one described for contact events above. Deaths of existing hosts/vectors can occur both naturally or due to infection lethality. Only deaths due to infection are tracked and recorded in the model's history.

De novo mutation of a pathogen in a given host/vector results in a single locus within a pathogen's genome being randomly assigned a new allele from the possible alleles at that position. Recombination of two pathogens in a given host/vector creates two new genomes based on the independent segregation of chromosomes (or reassortment of genome segments, as in influenza virus biology) from the two parent genomes. In addition, there may be a Poisson-distributed random number of crossover events between homologous parent chromosomes. Recombination by crossover event will result in all the loci in the chromosome on one side of the crossover event location being inherited from one of the parents, while the remainder of the chromosome is inherited from the other parent. The locations of crossover events are distributed throughout the genome following a uniform random distribution.

\section{Model interventions}

Furthermore, the user may specify changes in model behavior at specific timepoints during the simulation. These changes are known as "interventions". Interventions can include any kind of manipulation to populations in the model, including:

- $\quad$ adding new populations

- changing a population's event parameters and adaptive landscape functions

- linking and unlinking populations through migration or inter-population contact

- adding and removing hosts and vectors to a population

Interventions can also include actions that involve specific hosts or vectors in a given population, such as:

- $\quad$ adding pathogens with specific genomes to a host/vector

- removing all protection sequences from some hosts/vectors in a population

- applying a "treatment" in a population that cures some of its hosts/vectors of pathogens

- applying a "vaccine" in a population that protects some of its hosts/vectors from pathogen infection 
For these kinds of interventions involving specific pathogens in a population, the user may choose to apply them to a randomly-sampled fraction of hosts/vectors in a population, or to a specific group of individuals. This is useful when simulating consecutive interventions on the same specific group within a population. A single model may contain multiple groups of individuals and the same individual may be a member of multiple different groups. Individuals remain in the same group even if they migrate away from the population they were chosen in.

When a host/vector is given a "treatment", it removes all pathogens within the host/vector that do not contain a collection of sequence motifs called "resistance sequences". A treatment may have multiple resistance sequences. A pathogen must contain all of these within its genome in order to avoid being removed. On the other hand, applying a vaccine consists of adding a specific protection sequence to hosts/vectors, which behaves as explained above for recovered hosts/vectors when they acquire immune protection, in models that allow it.

\section{Model simulation}

Models are simulated using an implementation of the Gillespie algorithm in which the rates of different kinds of events across different populations are computed with each population's parameters and current state, and are then stored in a matrix. In addition, each population has host and vector matrices containing coefficients that represent the contribution of each host and vector, respectively, to the rates in the master model rate matrix (Fig. S1). Each coefficient is dependent on the genomes of the pathogens infecting its corresponding vector or host. In addition, the contribution of each pathogen genome is weighted by the share of total competitive fitness it holds within the host or vector. This occurs under the simplifying assumption that in a coinfection, pathogens with more competitive fitness will have higher intra-host (or intra-vector) shares of the pathogen population.

All $x$ populations in a model have matrices composed of coefficients $c$ for all $n$ event types and all $y_{h}$ hosts or $y_{v}$ vectors in the population. Hosts and vectors are handled in separate matrices. The coefficient $c$ for a given event $e$ and host $j_{h}$ (or vector $j_{v}$ ) in population $i$ is computed from the intra-host competitive fitness function $\varphi$ and the family of functions $f$, which return a value between 0 and 1 for all $z$ pathogen genomes $g$ inside the given host $j_{h}$ or vector $j_{v}$ at time $t$ :

$$
\begin{aligned}
& c_{i, j_{h}, e}= \begin{cases}\sum_{k=1}^{z_{j_{h}}} f_{i, h}\left(g_{k}, t\right) \cdot \phi_{i, h}\left(g_{k}, t\right) & z_{j_{h}}>0 \\
1 & z_{j_{h}}=0 \text { or } e \notin E\end{cases} \\
& c_{i, j_{v}, e}=\left\{\begin{array}{lll}
\sum_{k=1}^{z_{j v}} f_{i, v}\left(g_{k}, t\right) \cdot \phi_{i, v}\left(g_{k}, t\right) & z_{j_{v}}>0 & \text { or } \quad e \notin E \\
1 & z_{j_{v}}=0 & \text { and } e \in E
\end{array}\right.
\end{aligned}
$$




\section{$E=\{$ birth, receive contact, receive population contact, migration $\}$}

The values of $\varphi$ and $f$ are relative to a reference value of 1 . Functions $\varphi$ and $f$ are defined to be 1 for any given genome $g$ by default, but can be set to any arbitrary function that maps a genome sequence to a value between 0 and 1 . Each population may have different functions $\varphi$ and $f$ for hosts and vectors, and they may be different across populations and time points, as with any other parameter. Coefficients for hosts and vectors with no pathogens are by default zero, save for birth, receiving intra- or inter-population contact, and migration. In these cases, the events may still involve healthy individuals, and the coefficients are therefore equal to 1.

With these coefficients (in units of hosts or vectors for $c_{h}$ and $c_{v}$, respectively), the rates $r$ of each kind of event can now be calculated (in units of time ${ }^{-1}$ ). Some events concern a single host or vector, such as birth, death, mutation, and recombination rates. The total birth rates of hosts and vectors in a population $i$ are calculated from the population birth rates $\alpha$ (units of time ${ }^{-1}$ ) at time $t$ as

$$
\begin{gathered}
r_{i, h, e=\text { birth }}=\alpha_{i, h}(t) \sum_{j_{h}=1}^{y_{i, h}} c_{i, j_{h}, e=\text { birth }} \\
r_{i, v, e=\text { birth }}=\alpha_{i, v}(t) \sum_{j_{v}=1}^{y_{i, v}} c_{i, j_{v}, e=\text { birth }}
\end{gathered}
$$

The total natural death rates of hosts and vectors in a population $i$ are calculated from the population death rates $\gamma$ (units of time ${ }^{-1}$ ) at time $t$ as

$$
\begin{aligned}
& r_{i, h, e=\text { n.death }}=\gamma_{i, h}(t) y_{i, h} \\
& r_{i, v, e=\text { n.death }}=\gamma_{i, v}(t) y_{i, v}
\end{aligned}
$$

Death by natural causes is unaffected by infection, and thus only depends on the total number of hosts $y_{i, h}$ or vectors $y_{i, v}$. Death due to pathogen infection is counted separately in order to better track the system's epidemiology. The total lethality rates for hosts and vectors in a population $i$ are calculated from the population-specific case fatality rates $\tau$ (a dimensionless fraction) and recovery rates $\delta$ (which determine the mean infection durations, $1 / \delta$; $\delta$ units of time $^{-1}$ ) at time $t$ as

$$
r_{i, h, e=\text { lethality }}=\tau_{i, h}(t) \cdot \delta_{i, h}(t) \sum_{j_{h}=1}^{y_{i, h}} c_{i, j_{h}, e=\text { lethality }}
$$




$$
r_{i, v, e=\text { lethality }}=\tau_{i, v}(t) \cdot \delta_{i, v}(t) \sum_{j_{v}=1}^{y_{i, v}} c_{i, j_{v}, e=\text { lethality }}
$$

The total recovery rates of hosts and vectors of a population $i$ are calculated based on population-specific recovery rates $\delta$ (units of time ${ }^{-1}$ ) at time $t$ as

$$
\begin{gathered}
r_{i, h, e=\text { recovery }}=\delta_{i, h}(t) \sum_{j_{h}=1}^{y_{i, h}} c_{i, j_{h}, e=\text { recovery }} \\
r_{i, v, e=\text { recovery }}=\delta_{i, v}(t) \sum_{j_{v}=1}^{y_{i, v}} c_{i, j_{v}, e=\text { recovery }}
\end{gathered}
$$

The total rates of de novo mutation in hosts and vectors of a population $i$ are calculated based on population-specific mutation rates $\mu$ (units of time ${ }^{-1}$ ) at time $t$ as

$$
\begin{gathered}
r_{i, h, e=\text { mutation }}=\mu_{i, h}(t) \sum_{j_{h}=1}^{y_{i, h}} c_{i, j_{h}, e=\text { mutation }} \\
r_{i, v, e=\text { mutation }}=\mu_{i, v}(t) \sum_{j_{v}=1}^{y_{i, v}} c_{i, j_{v}, e=\text { mutation }}
\end{gathered}
$$

Similarly, the total rates of recombination in hosts and vectors of a population $i$ are calculated based on population-specific recombination rates $\rho$ (units of time ${ }^{-1}$ ) at time $t$ as

$$
\begin{aligned}
r_{i, h, e=\text { recombination }} & =\rho_{i, h}(t) \sum_{j_{h}=1}^{y_{i, h}} c_{i, j_{h}, e=\text { recombination }} \\
r_{i, v, e=\text { recombination }} & =\rho_{i, v}(t) \sum_{j_{v}=1}^{y_{i, v}} c_{i, j_{v}, e=\text { recombination }}
\end{aligned}
$$

Some events involve two individuals $j_{1}$ and $j_{2}$ within the same population. The intra-population contact rates for host-host, host-vector, and vector-host contact in a population $i$ are calculated based on population-specific contact rates $\beta$ (units of time ${ }^{-1}$ for host-host transmission, time $^{-1}$ host/vector for vector-borne transmission) and transmission efficiency $\varepsilon$ (dimensionless fraction) at time $t$ as 


$$
\begin{gathered}
r_{i, h, e=\text { c.host-host }}=\epsilon_{i, h h}(t) \beta_{i, h}(t) \frac{\sum_{j_{h 1}=1}^{y_{i, h}} p_{i, j_{h 1}} c_{i, j_{h 1}, e=\text { transmit }}}{y_{i, h}} \sum_{j_{h 2}=1}^{y_{i, h}} c_{i, j_{h 2}, e=\text { receive }} \\
r_{i, h, e=\text { c.host-vector }}=\epsilon_{i, h v}(t) \beta_{i, v}(t) \frac{\sum_{j_{h}=1}^{y_{i, h}} p_{i, j_{h}} c_{i, j_{h}, e=\text { transmit }}}{y_{i, h}} \sum_{j_{v}=1}^{y_{i, v}} c_{i, j_{v}, e=\text { receive }} \\
r_{i, v, e=\text { c.vector-host }}=\epsilon_{i, v h}(t) \beta_{i, v}(t) \sum_{j_{v}=1}^{y_{i, v}} p_{i, j_{v}} c_{i, j_{v}, e=\text { transmit }} \frac{\sum_{j_{h}=1}^{y_{i, h}} c_{i, j_{h}, e=\text { receive }}}{y_{i, h}}
\end{gathered}
$$

Variable $p$ tracks whether a given host or vector is infected with pathogens $(p=1)$ or not $(p=0)$. Note that for host-vector and vector-host contact, $\beta$ corresponds to $\beta_{v}$ and is given in units of time $^{-1}$ host/vector. This follows the convention of defining contact rate in terms of the vector biting rate for parasitic vectors.

Some events involve two different populations. The migration rates of hosts and vectors in a population are calculated based on migration rates $\theta$ (units of time ${ }^{-1}$ ) from a specific population $i$ to each of all of its $q$ possible migration neighbors at time $t$ as

$$
\begin{aligned}
r_{i, h, e=\text { migration }} & =\sum_{l=1}^{q_{i, h}} r_{i l, h, e=\text { migration }} \\
= & \sum_{l=1}^{q_{i, h}} \theta_{i l, h}(t) \sum_{j_{h}=1}^{y_{i, h}} c_{i, j_{h}, e=\text { migration }} \\
r_{i, v, e=\text { migration }} & =\sum_{l=1}^{q_{i, v}} r_{i l, v, e=\text { migration }}=\sum_{l=1}^{q_{i, v}} \theta_{i l, v}(t) \sum_{j_{v}=1}^{y_{i, v}} c_{i, j_{v}, e=\text { migration }}
\end{aligned}
$$

Finally, some events involve two individuals in different populations. The inter-population contact rates for host-host, host-vector, and vector-host contact are calculated based on population-specific contact rates $\kappa$ (units of time ${ }^{-1}$ for host-host transmission, time ${ }^{-1}$ host/vector for vector-borne transmission) from a specific population $i$ to each of all of its $u$ possible contact neighbors at time $t$ as

$$
\begin{aligned}
r_{i, h, e=\text { p.c.host }- \text { host }} & =\sum_{l=1}^{u_{i, h}} r_{i l, h, e=\text { p.c.host }- \text { host }} \\
& =\sum_{l=1}^{u_{i, h}} \kappa_{i l, h h}(t) \kappa_{l i, h h}(t) \frac{\sum_{j_{h 1}=1}^{y_{i, h}} p_{i, j_{h 1}} c_{i, j_{h 1}, e=\text { p.c.transmit }}}{y_{i, h 1}} \sum_{j_{h 2}=1}^{y_{l, h}} c_{l, j_{h 2}, e=\text { p.c.receive }}
\end{aligned}
$$




$$
\begin{aligned}
r_{i, h, e=\text { p.c.host-vector }} & =\sum_{l=1}^{u_{i, h}} r_{i l, h, e=\text { p.c.host-vector }} \\
& =\sum_{l=1}^{u_{i, h}} \kappa_{i l, h v}(t) \kappa_{l i, v h}(t) \frac{\sum_{j_{h}=1}^{y_{i, h}} p_{i, j_{h}} c_{i, j_{h}, e=\text { p.c.transmit }}}{y_{i, h}} \sum_{j_{v}=1}^{y_{l, v}} c_{l, j_{v}, e=\text { p.c.receive }} \\
r_{i, h, e=\text { p.c. vector-host }} & =\sum_{l=1}^{u_{i, v}} r_{i l, h, e=\text { p.c.vector-host }} \\
& =\sum_{l=1}^{u_{i, v}} \kappa_{i l, v h}(t) \kappa_{l i, h v}(t) \sum_{j_{v}=1}^{y_{i, v}} p_{i, j_{v}} c_{i, j_{v}, e=\text { p.c.transmit }} \frac{\sum_{j_{h}=1}^{y_{l, h}} c_{l, j_{h}, e=\text { p.c.receive }}}{y_{l, h}}
\end{aligned}
$$

With the rates for all events and populations computed, an event and population is selected randomly, with probabilities drawn from all relative rates as calculated above. In the case of events that involve two populations, the second population is chosen randomly, this time with probabilities drawn from the rates of migration or population contact specifically originating from the first selected population. In similar fashion, the individual host(s) and/or vector(s) are sampled randomly based on the corresponding coefficients within the chosen population(s). Finally, the individual pathogen genomes are sampled randomly based on their respective contributions to the chosen host/vector coefficient. As explained above, mutation events sample a single pathogen, recombination events sample two pathogens, and contact events sample a Poisson-distributed random number of pathogens with replacement. The mean of this distribution is the mean inoculum size parameter, $n_{i}$. Whenever an event occurs, the corresponding coefficients for the hosts and vectors affected in the population matrices are updated, and the master rate matrix is recomputed based on this information.

The model's state at any given time comprises all populations, their hosts and vectors, and the pathogen genomes infecting each of these. A copy of the model's state is saved at every time point, or at intermittent intervals throughout the course of the simulation. A random sample of hosts and/or vectors may be saved instead of the entire model as a means of reducing memory footprint.

\section{Model output}

The output of a model can be saved in multiple ways. The model state at each saved time point may be output in a single data frame and saved as a tabular file. Other data output types include counts of pathogen genomes or protection sequences for the model, as well as time of first emergence for each pathogen genome and genome distance matrices for every time point sampled. 
The user can also create different kinds of plots to visualize the results. These include:

- plots of the number of hosts and/or vectors in different epidemiological compartments (naive, infected, recovered, and dead) across simulation time

- plots of the number of individuals in a compartment for different populations

- plots of the genomic composition of the pathogen population over time

- phylogenies of pathogen genomes

Users can also use the data output formats to make their own custom plots.

\section{Models used in this study}

We used the Opqua modeling framework to study the evolutionary dynamics of pathogens across low fitness regimes. The code to run all simulations and analyses presented in this work is available in a separate GitHub repository (github.com/pablocarderam/fitness_valleys_opqua). The simulations were run on a 2019 MacBook Pro laptop with a $2.4 \mathrm{GHz} 8$-Core Intel Core i9 processor.

\section{Stochastic models of fitness valleys}

To simulate evolution across a fitness valley (Fig. 2), we constructed a stochastic host-host transmission model using Opqua. The model comprises 1000 hosts in a single population, 500 of which start the simulation infected by "wild-type” pathogens with a genome of "AAAAAAAA". The epidemiological parameters were set to the default values shown for host-host models in Table S1, with the following modifications: a genome length of 8 loci with two possible alleles ("A" and "B") at each locus, a mean inoculum of 1 pathogen, a recovery rate of 0.0051 /unit time, a mutation rate of 0.021 /unit time, and no recombination. The recovery rate of "resistant" pathogens was set to be $75 \%$ of the original for pathogens with a genome of "BBBBBBBB”. The choice of using bi-allelic rather than multi-allelic loci (such as four DNA bases, 20 amino acids, or other kinds of allelic variations) was made for simplicity and to more easily observe stochastic tunneling. In systems with many more possible alleles, the probability of reaching a specific other genotype is exponentially lower, and therefore the number of simulation replicates needed to observe differences in stochastic tunneling rates rises exponentially as well.

Most importantly, the intra-host competitive fitness $\varphi$ of each genome was made to follow the following function, describing a fitness valley in terms of the number of " $\mathrm{B}$ " alleles in the genome $b$ and the total length of the genome $g$ : 


$$
\phi(b, g)= \begin{cases}\frac{1}{2(b+1)^{5}} & x<3 \\ \frac{1}{(g-b+1)^{3}} & x \geq 3\end{cases}
$$

This function was chosen as it describes a steep initial decrease in fitness as pathogens mutate away from the wild type, followed by a more gradual increase in fitness to the resistant genotype. It also guarantees that all mutant pathogens have lower competitive fitness than the wild-type, save for the resistant mutant genome, which outcompetes even the wild type. In this way, resistant pathogens fixate in the population more easily once they arise, which facilitates observation of the stochastic tunneling phenomena being studied.

Additionally, a "drug treatment" intervention was added at time 10,000 such that all pathogens with genomes other than the resistant genotype were cleared from the population.

The simulations shown in Fig. 2E aims to illustrate the evolution of strictly less-fit mutants in the system. Therefore, the previous setup was modified to finish simulation at 10000 time units with no drug intervention, and the contact and mutation rates of resistant pathogens were modified to be equal to zero. This effectively makes the resistant genotype into a lethal mutation, allowing easier study of the remainder of less-fit genotypes without interference. To study the distribution of survival times for mutants that are alone before they become coinfected by a wild-type pathogen or are cleared from the host, we extracted the survival times for 100 mutant-only infections in a single simulation run for each contact rate.

To evaluate the effect of different kinds of fitness valleys (Fig. S2), we varied the number of loci being taken into account for fitness (thus varying the genome length $g$ and the length of the fitness valley) as well as $a_{d}$, the fraction of the original fitness drop with respect to the wild-type genome (thus varying the minimum fitness of mutants, or the depth of the valley). This was done with the following fitness function:

$$
\phi\left(b, g, a_{d}\right)= \begin{cases}\frac{1}{2}\left[\frac{1-a_{d}}{(b+1)^{5}}+a_{d}\right] & x<\min (3, g) \\ \frac{1}{2}\left[\frac{2-a_{d}}{(g-b+1)^{3}}+a_{d}\right] & x \geq \min (3, g)\end{cases}
$$

\section{Compartment model of mutant and wild-type pathogen competition}

To study the competitive dynamics of wild-type pathogens and mutant pathogens with lower fitness, we developed a compartment model describing the number of hosts infected with no pathogens $(S)$, wild-type pathogens only $(W)$, mutant pathogens only $(M)$, or coinfected with both kinds of pathogens $(C)$. This model is presented in Fig. 3A, and shown in more detail in Fig. S3. The system considers a constant host population $N$ such that $N=S+M+W+C$. The 
following system of ordinary differential equations (ODEs) describes the flow between host compartments in terms of host recovery rate $\delta$, contact rate $\beta$, mutation rates from wild-type to mutants $\mu_{1}$ and vice-versa $\mu_{2}$, inoculum size $n_{i}$, and the probability of wild-type pathogens with higher fitness outcompeting mutants in intra-host competition $\psi$ :

$$
\left\{\begin{aligned}
\frac{d S}{d t} & =\left(\delta-\beta \frac{S}{N}\right)(N-S) \\
\frac{d W}{d t} & =\frac{\beta}{N}\left[W\left(S-M-\left[1-\psi^{n_{i}}\right] C\right)+\psi^{n_{i}} S C\right]-\left(\delta+\mu_{1}\right) W \\
\frac{d M}{d t} & =\frac{\beta}{N}\left[M\left(S-W-\left[1-(1-\psi)^{n_{i}}\right] C\right)+(1-\psi)^{n_{i}} S C\right]-\left(\delta+\mu_{2}\right) M \\
\frac{d C}{d t} & \left.=\frac{\beta}{N}\left[C\left(\left[1-\psi^{n_{i}}-(1-\psi)^{n_{i}}\right)\right] S+\left(1-\psi^{n_{i}}\right) W+\left[1-(1-\psi)^{n_{i}}\right] M\right)+2 W M\right] \\
& +\mu_{1} W+\mu_{2} M-\delta C
\end{aligned}\right.
$$

For models in which only a single mutation is possible such as the model above, the relationship between the probability parameter $\psi$ used in the ODE model, the relative intra-host competitive fitness $\varphi$ used in Opqua models, and the fitness disadvantage of mutation $\lambda$ shown on Figs. 3 and $S 4$ is given by

$$
\psi=\frac{1}{2-(1-\phi)}=\frac{1}{2-\lambda}
$$

Solving the model at equilibrium leads to $S^{*}=\delta N / \beta$ for all nontrivial solutions, but the remaining three steady-state compartments yield unwieldy expressions that are difficult to analyze.

We numerically solved the deterministic behavior of this model using the parameters described in Table S3 from starting conditions of 10 infected individuals, taking the system state after 1000 time units as a result. Mutations that restore the exact wild-type genotype were assumed to be far less likely than mutations away from the genotype $\left(\mu_{1}<<\mu_{2}=0\right)$.

\section{Stochastic model of pathogen competition in a descending fitness landscape}

To establish the potential of genomic epidemiological models as tools to study evolution, we created a stochastic model using Opqua following and expanding our results from the compartment model (Fig. 3). The model is composed of 500 hosts in a single population, 250 of which start the simulation infected by “wild-type” pathogens with a specific genome sequence 
of 500 amino acids. Each simulation lasted for 1000 time units, and the result shown for each condition was obtained as the mean of ten replicates. The epidemiological parameters were set to the default values shown for host-host models in Table S1, with the following modifications: a genome length of 500 loci with 20 possible alleles (corresponding to the 20 amino acids abbreviations "ARNDCEQGHILKMFPSTWYV”) at each locus, a mean inoculum of 1 pathogen, a mutation rate of 0.051 /unit time, and no recombination. The full amino acid alphabet was used in this model to showcase the capabilities of Opqua and genomic epidemiological modeling as a way of describing complex diversity. Since this simulation does not aim to show stochastic tunneling to a specific new fitness peak, all evolutionary trajectories away from wild type are considered of interest, allowing for the use of more complex genomes and allele systems.

The intra-host competitive fitness $\varphi$ of each genome was made to follow the following function, describing an exponential decay in fitness in terms of the relative fitness disadvantage of each successive mutation $\lambda$ (shown on Fig. 3 ) and the Hamming distance $d$ between a mutant genome to the wildtype genome:

$$
\phi(d, \lambda)=(1-\lambda)^{d}
$$

The maximum distance between a mutant genome and wild-type and the mean pairwise distance between genomes at the end of simulation were both computed as Hamming distances.

\section{Stochastic model of population determinants of evolution}

We then used the same modeling framework to examine the effect of population size and migration between populations on evolution across descending fitness landscapes (Fig. 4). The parameters and analyses used were the same as the ones used for the previous stochastic model describing a descending fitness landscape, unless otherwise noted on each figure. The host-host contact rate was fixed to $\beta=0.1251 /$ unit time, and the fitness cost of each successive mutation was set to $\lambda=0.5$. For the metapopulation model, the system consisted of 10 different interconnected populations of 10 initial hosts each.

\section{Stochastic model of pathogen determinants of evolution}

Finally, to study the effect of different biological factors on evolution across descending fitness landscapes, we modeled a host-vector system using Opqua (Fig. 5). The model consisted of 250 hosts and 250 vectors in a single population, running for 1000 time units in 10 replicates for each condition. Model parameters were set to the default values shown on Table S3, with a few modifications. All simulations had the host-vector contact rate set to $0.1251 /$ unit time. The fitness cost of each successive mutation was set to $\lambda=0.5$ within hosts only, and was set to $\lambda=0$ 
within vectors, to model the differing selection pressures on the genome in each organism. Simulations which varied recombination rates had a mean number of crossover events in both hosts and vectors set to 5. Other variables were set as shown on Fig. 5, or the default values on Table S2 if not noted.

An analogous set of simulations was repeated in a host-host transmission system as shown in Figs. S5 and S6. All parameters were identical as in the host-vector system described, except for the host-host contact rate set to 0.1251 /unit time and the host-vector contact rate set to zero, as well as the parameters indicated on each figure.

Figures S1 - S6 


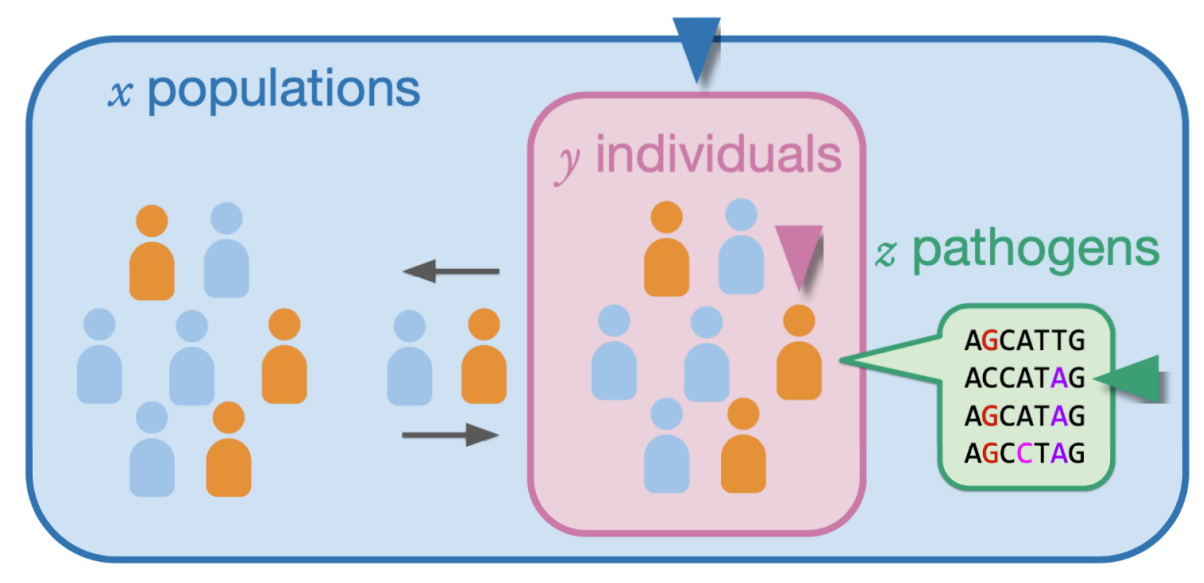

A n events

B

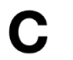

$n$ events

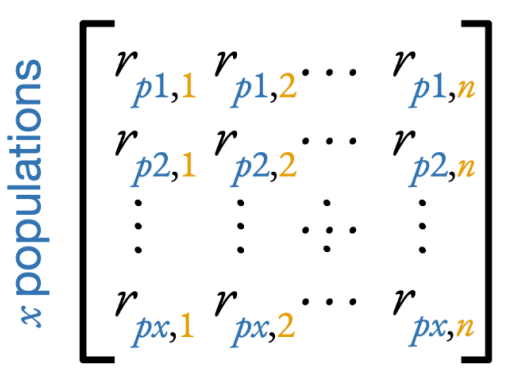

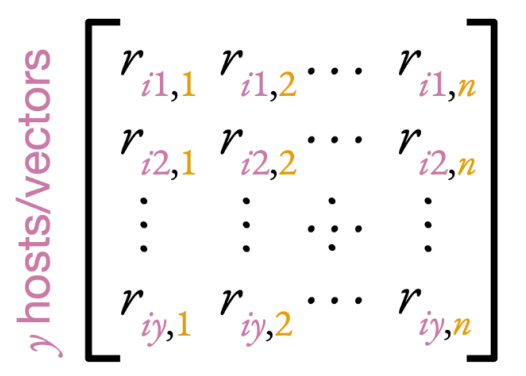

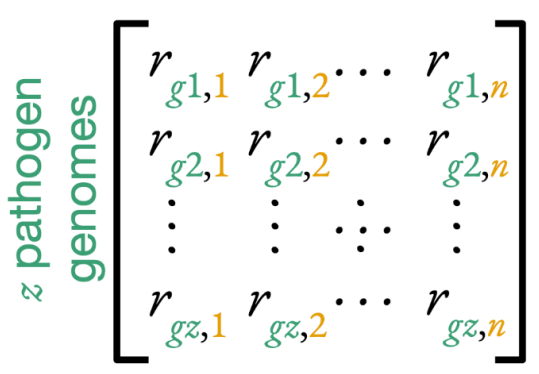

Supplementary Fig. S1. Opqua uses different levels of rate matrices to sample events. (A) A matrix contains the rates $r_{p}$ of $n$ different events for $x$ different populations within the model. A given event type and population are sampled randomly based on the rate of each event-population combination. (B) Each population contains matrices with the event rates $r_{i}$ for all $y$ individual hosts and vectors it contains. A host/vector is sampled randomly based on the rates of the chosen event type for all host/vectors in the chosen population. (C) Finally, a similar process occurs within hosts and vectors to randomly sample pathogens within them, based on how their $z$ different genomes affect the rates $r_{g}$ of the chosen event. Some events involve sampling an additional population (migration or inter-population contact), host/vector (inter- or intra-population contact), or pathogen (recombination). Once event type, population(s), host(s) and/or vector(s), and pathogen(s) have been chosen in this manner, the state of the model is adjusted according to the event, and the relevant rate changes are propagated upward from within the pathogens affected to the host(s), vector(s), populations, and overall model they are in. 
A
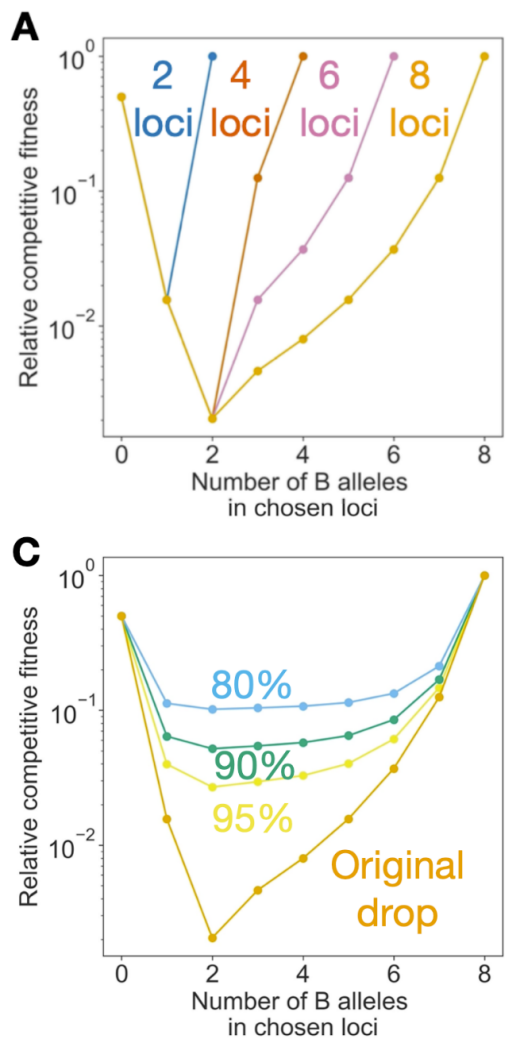

B

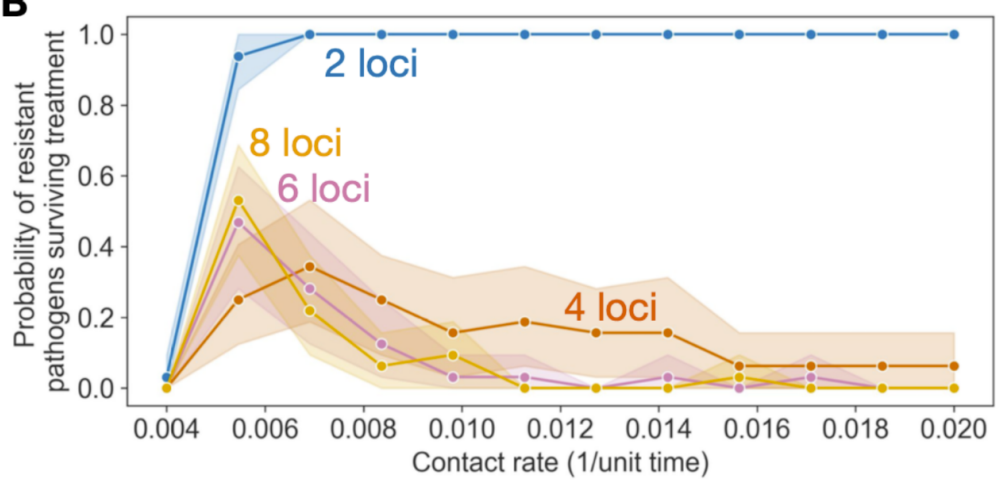

D

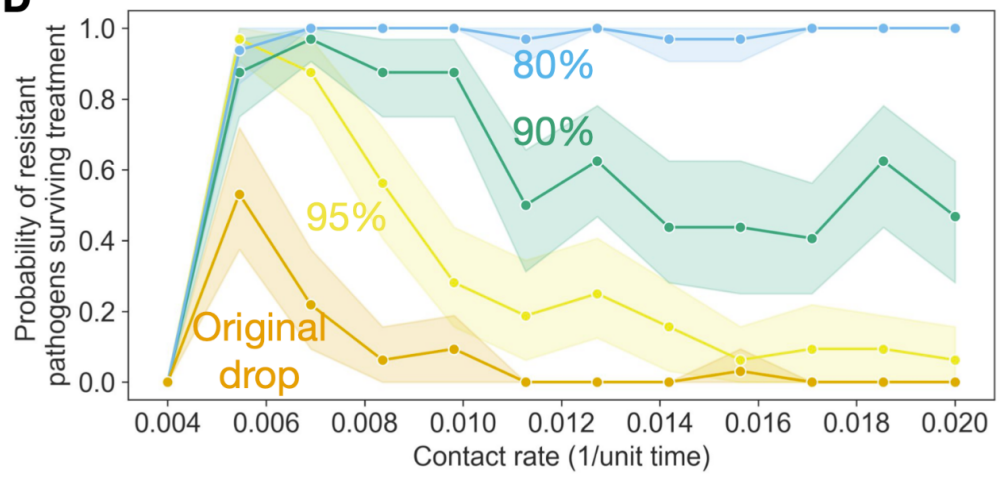

Supplementary Fig. S2. Length and steepness of the fitness valley affect the impact of competition on evolution. We constructed a model to simulate stochastic tunneling of a pathogen across a fitness valley based on genomes with eight bi-allelic loci, as portrayed in Fig.

2. (A) By focusing on a subset of loci in each genome, we can vary the length of the fitness valley. (B) Longer fitness valleys favor the evolution and survival of pathogens in low transmission environments, as more loci provide more evolutionary paths through the valley but require little intra-host competition. In short fitness valleys, the number of paths becomes more restricted while the effect of competition is lessened, favoring environments with increased transmission. (C) By adjusting the fitness cost of intermediate mutants, we can vary the steepness and depth of the fitness valley. (D) Steep, deep valleys increase the effect of intra-host competition, favoring the evolution and survival of pathogens in low transmission environments more heavily. 
A
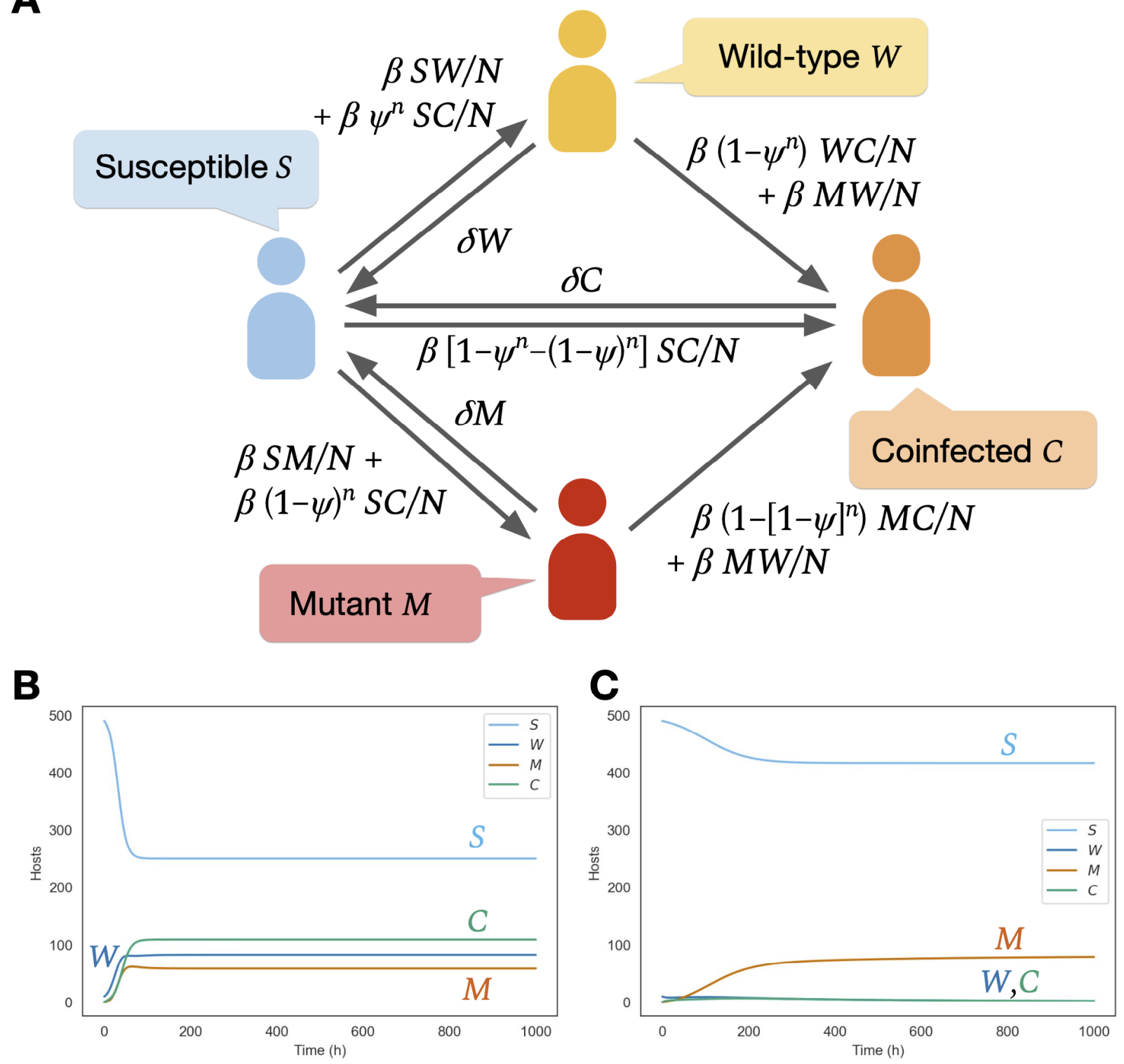

Supplementary Fig. S3. Compartment model structure allows deterministic simulation of dynamics of two strains of pathogens. (A) The model consists of compartments for hosts infected with no pathogens $(S)$, wild-type pathogens only $(W)$, mutant pathogens only $(M)$, or coinfected with both kinds of pathogens $(C)$. Transitions between compartments are determined by host recovery rate $\delta$, contact rate $\beta$, mutation rates from wild-type to mutants $\mu_{1}$ and vice-versa $\mu_{2}$, inoculum size $n$, and the probability of wild-type pathogens with higher fitness outcompeting mutants in intra-host competition $\psi$. (B) At high transmission intensities $(\beta=0.2)$, unfit mutants $(\psi=0.67)$ constitute a small portion of the total population, as shown throughout the rest of this work. (C) At low transmission intensities $(\beta=0.12)$, unfit mutants show greater prevalence in the population. 

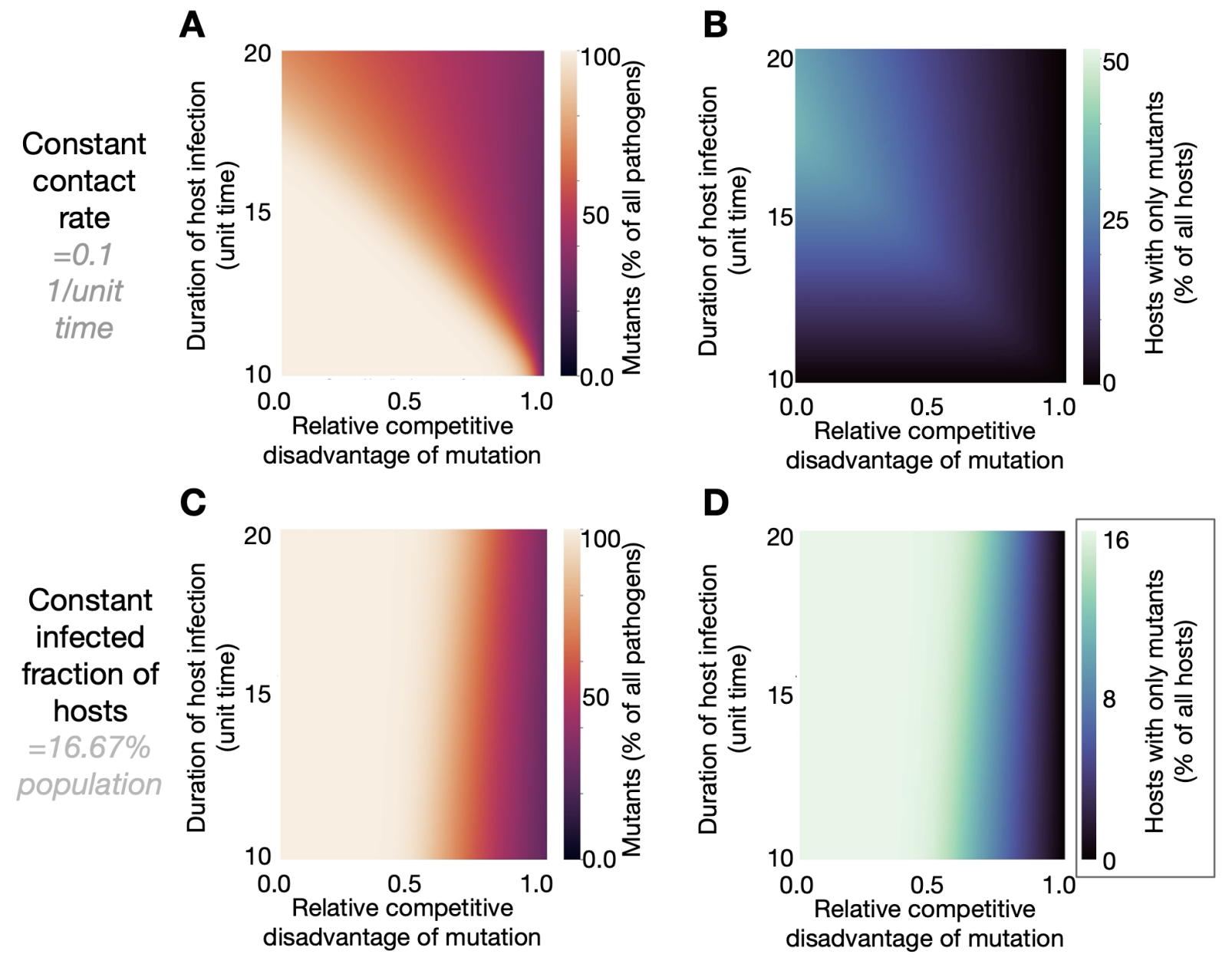

\footnotetext{
Supplementary Fig. S4. Duration of infection affects mutant fraction primarily through competition for free hosts, as with contact rate. Using the ordinary differential equation model described, we vary the duration of infection (equivalent to 1/recovery rate) while keeping the contact rate constant such that the range of steady-state infected hosts sampled is equivalent to those in Figure 3B and 3C. The resulting (A) mutant fractions of pathogens and (B) mutant-only fractions of infections are similar to those shown when varying contact rates in Figure 3B and 3C. Small differences in the scale of the effects are due to the fact that at high durations of infection, mutants are removed less frequently through recovery. This can be seen when varying the duration of infection while keeping the fraction of infected hosts constant by simultaneously varying the contact rate. While biologically unlikely to happen, this shows a small increase in fraction of (C) mutants among pathogens and (D) mutant-only infections among hosts (note change in color scale).
} 

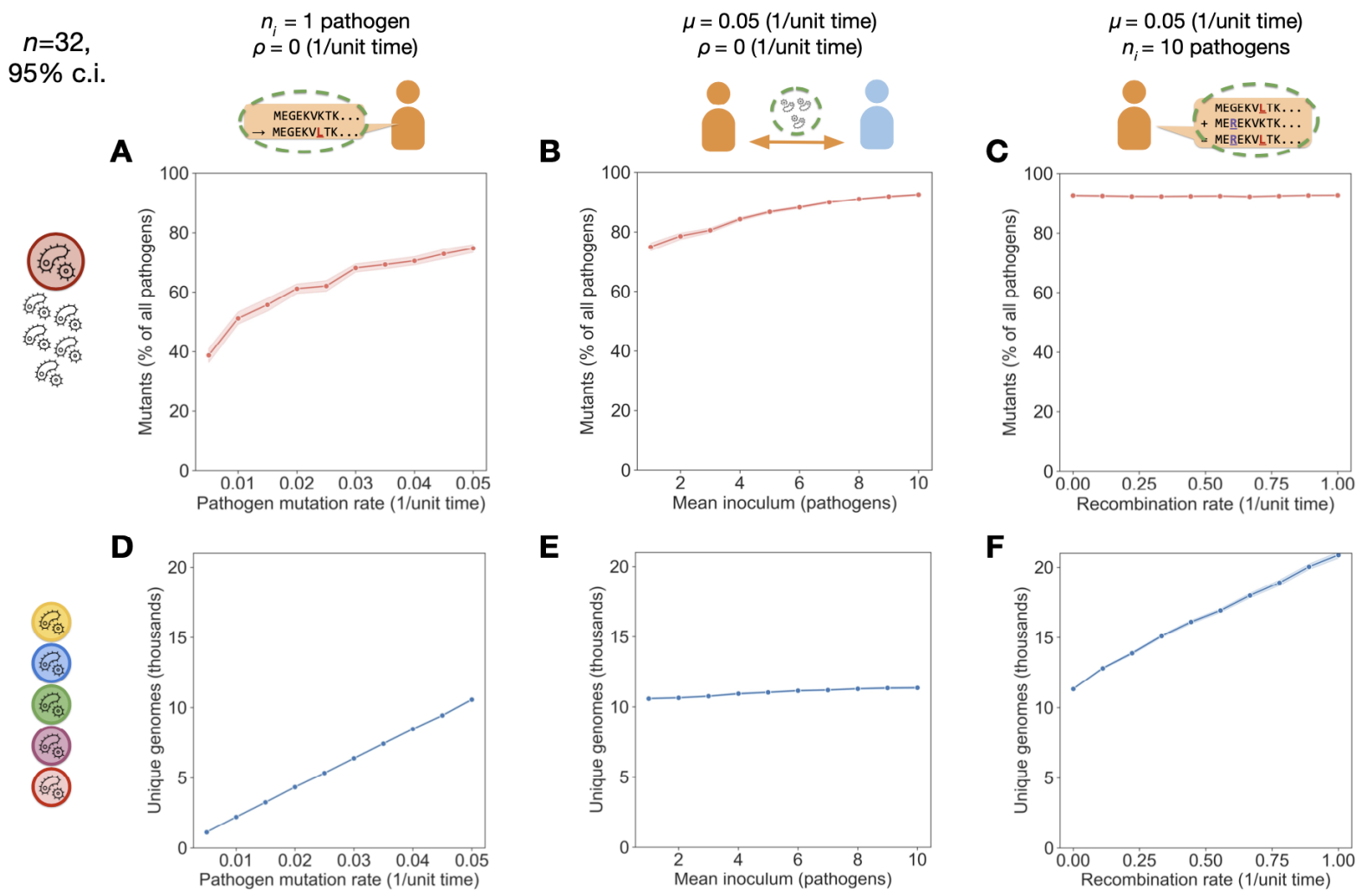

E

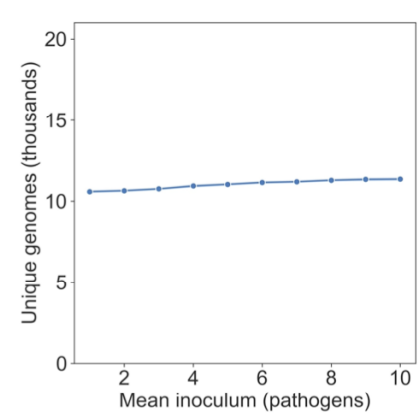

H

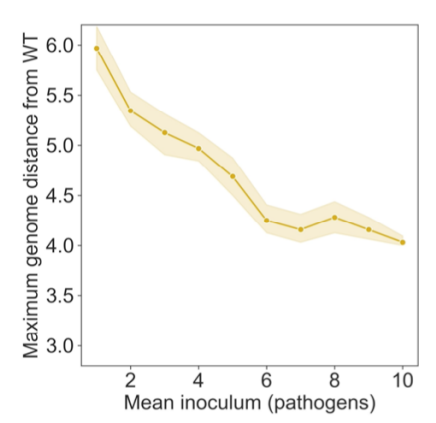

$\mathbf{F}$
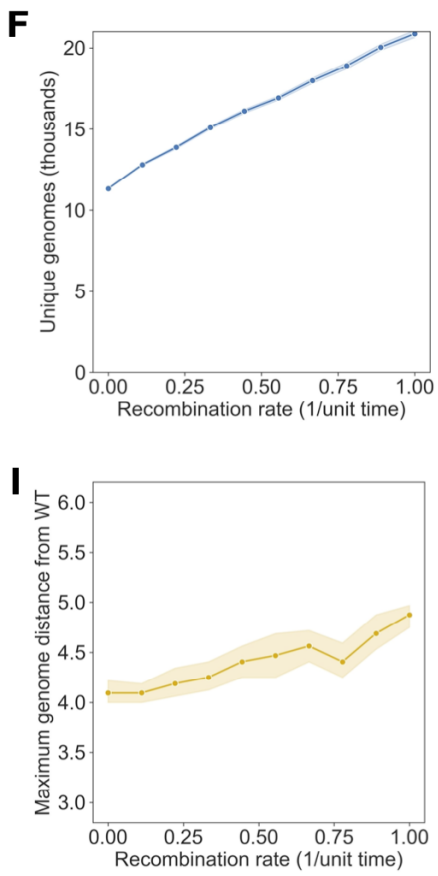

\section{Supplementary Fig. S5. Pathogen biology affects the distribution and dimensions of} their evolution for host-host direct transmission. The effects of mutation rate, inoculum size from hosts, and recombination rates are analogous to those observed within hosts for a vector-borne model (Fig. 5). The fraction of mutants in the pathogen population is increased by both (A) high mutation rates $(\mu)$ and (B) high mean inoculum sizes $\left(n_{i}\right)$, but is unaffected by (C) recombination rates $(\rho)$. (D) The number of unique pathogen genomes in the simulation, which we treat as a measure of the "width" of evolutionary space explored, increases with high mutation rates, but less so with (E) high mean inoculum size. (F) Greater recombination increases the number of unique pathogen genomes. Lastly, (G) increased mutation rate increases the "depth" of evolutionary space explored by pathogens, measured as the maximum 
bioRxiv preprint doi: https://doi.org/10.1101/2021.12.16.473045; this version posted December 17,2021 . The copyright holder for this preprint (which was not certified by peer review) is the author/funder, who has granted bioRxiv a license to display the preprint in perpetuity. It is made available under aCC-BY 4.0 International license.

Hamming distance of mutant genomes from the initial wild-type sequence. (H) Low inoculum size increases the probability of transmitting mutants without wild-type (WT) competitors, allowing for greater depth in the evolutionary space explored. (I) High recombination rates increase depth of evolutionary space, notably without affecting the fraction of mutants in the pathogen population. 

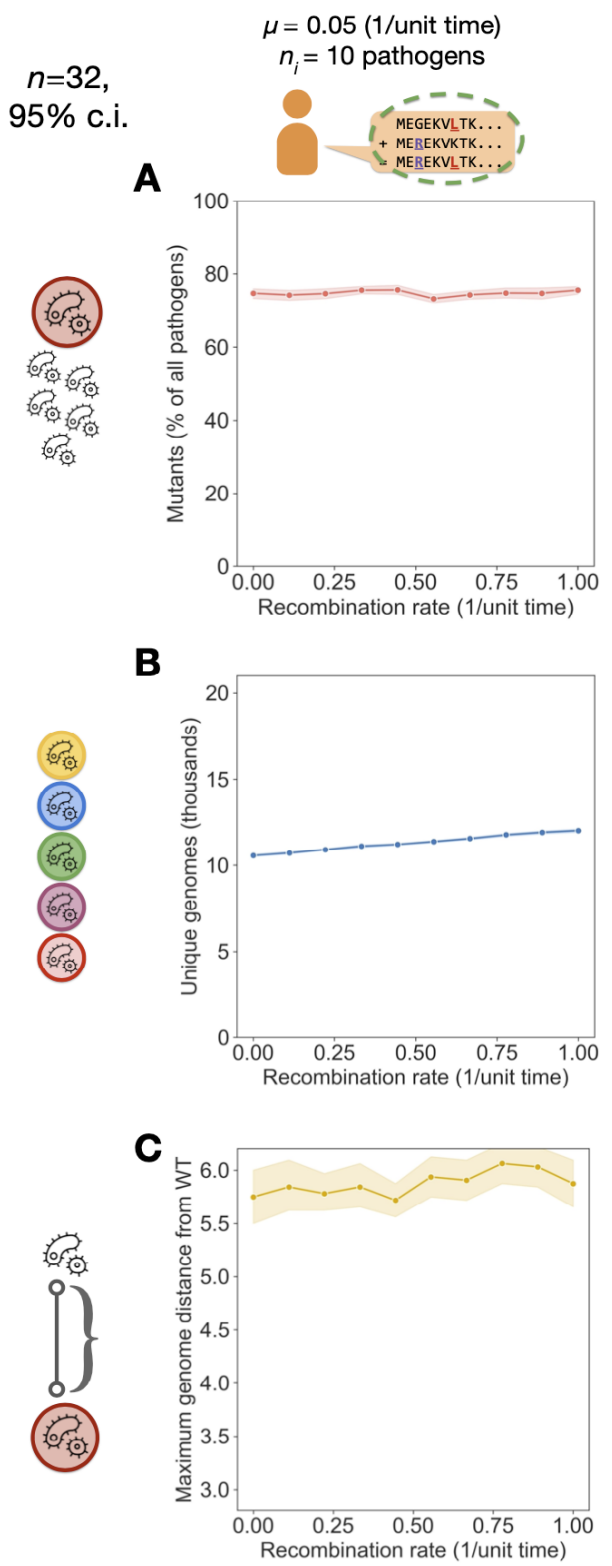

\section{Supplementary Fig. S56. Recombination depends on inoculum size to increase}

evolutionary distance. By keeping the mutation rate $(\mu)$ constant and reducing the mean inoculum size $\left(n_{i}\right)$ to 1 in a host-host transmission model with a descending fitness landscape, we can see the effects of recombination on pathogen genome evolution with low inoculums. (A) As before, increasing recombination rate does not increase the mutant fraction of pathogens. However, the lower inoculum greatly reduces the effect of recombination on (B) the number of unique pathogen genomes explored and (C) the maximum Hamming distance from the wild-type pathogen genotype. Neither of these shows increases on the same scale seen for the higher mean inoculum size $n_{i}=10$ in Fig. S3. 
Tables S1 - S3

Table S1: Default Opqua parameters for host-host transmission models*

\begin{tabular}{|l|l|}
\hline Parameter name & Default value \\
\hline num_loci & 10 \\
\hline possible_alleles & 'ATCG' \\
\hline fitnessHost & (lambda g: 1 ) \\
\hline contactHost & (lambda g: 1 ) \\
\hline receiveContactHost & (lambda g: 1 ) \\
\hline lethalityHost & (lambda g: 1 ) \\
\hline natalityHost & (lambda g: 1 ) \\
\hline recoveryHost & (lambda g: 1 ) \\
\hline migrationHost & (lambda g: 1 ) \\
\hline populationContactHost & (lambda g: 1 ) \\
\hline receivePopulationContactHost & (lambda g: 1 ) \\
\hline mutationHost & (lambda g: 1 ) \\
\hline recombinationHost & (lambda g: 1 ) \\
\hline fitnessVector & (lambda g: 1 ) \\
\hline contactVector & (lambda g: 1 ) \\
\hline receiveContactVector & (lambda g: 1 ) \\
\hline lethalityVector & (lambda g: 1 ) \\
\hline natalityVector & (lambda g: 1 ) \\
\hline recoveryVector & (lambda g: 1 ) \\
\hline migrationVector & \\
\hline
\end{tabular}




\begin{tabular}{|l|l|}
\hline populationContactVector & (lambda g: 1) \\
\hline receivePopulationContactVector & (lambda g: 1) \\
\hline mutationVector & (lambda g: 1) \\
\hline recombinationVector & (lambda g: 1) \\
\hline contact_rate_host_vector & 0 \\
\hline transmission_efficiency_host_vector & 0 \\
\hline transmission_efficiency_vector_host & 0 \\
\hline contact_rate_host_host & $2.00 \mathrm{E}-01$ \\
\hline transmission_efficiency_host_host & 1 \\
\hline mean_inoculum_host & $1.00 \mathrm{E}+01$ \\
\hline mean_inoculum_vector & 0 \\
\hline recovery_rate_host & $1.00 \mathrm{E}-01$ \\
\hline recovery_rate_vector & 0 \\
\hline lethality_rate_host & 0 \\
\hline lethality_rate_vector & 0 \\
\hline recombine_in_host & $1.00 \mathrm{E}-04$ \\
\hline recombine_in_vector & 0 \\
\hline num_crossover_host & 1 \\
\hline num_crossover_vector & 0 \\
\hline mutate_in_host & $1.00 \mathrm{E}-06$ \\
\hline mutate_in_vector & 0 \\
\hline death_rate_host & 0 \\
\hline
\end{tabular}




\begin{tabular}{|l|l|}
\hline death_rate_vector & 0 \\
\hline birth_rate_host & 0 \\
\hline birth_rate_vector & 0 \\
\hline vertical_transmission_host & 0 \\
\hline vertical_transmission_vector & 0 \\
\hline inherit_protection_host & 0 \\
\hline inherit_protection_vector & 0 \\
\hline protection_upon_recovery_host & None \\
\hline protection_upon_recovery_vector & None \\
\hline
\end{tabular}

*For a detailed description of each parameter, consult the documentation at https://github.com/pablocarderam/opqua\#newsetup

Table S2: Default Opqua parameters for vector-borne transmission models*

\begin{tabular}{|l|l|}
\hline Parameter name & Default value \\
\hline num_loci & 10 \\
\hline possible_alleles & 'ATCG' \\
\hline fitnessHost & (lambda g: 1 ) \\
\hline contactHost & (lambda g: 1 ) \\
\hline receiveContactHost & (lambda g: 1 ) \\
\hline lethalityHost & (lambda g: 1 ) \\
\hline natalityHost & (lambda g: 1 ) \\
\hline recoveryHost & (lambda g: 1) \\
\hline migrationHost & (lambda g: 1) \\
\hline populationContactHost & (lambda g: 1) \\
\hline
\end{tabular}




\begin{tabular}{|c|c|}
\hline receivePopulationContactHost & (lambda g: 1) \\
\hline mutationHost & (lambda g: 1) \\
\hline recombinationHost & (lambda g: 1) \\
\hline fitnessVector & (lambda g: 1) \\
\hline contactVector & (lambda g: 1) \\
\hline receiveContactVector & (lambda g: 1) \\
\hline lethalityVector & (lambda g: 1) \\
\hline natalityVector & (lambda g: 1) \\
\hline recoveryVector & (lambda g: 1) \\
\hline migrationVector & (lambda g: 1) \\
\hline populationContactVector & (lambda g: 1) \\
\hline receivePopulationContactVector & (lambda g: 1) \\
\hline mutationVector & (lambda g: 1) \\
\hline recombinationVector & (lambda g: 1) \\
\hline contact_rate_host_vector & $2.00 \mathrm{E}-01$ \\
\hline transmission_efficiency_host_vector & 1 \\
\hline transmission_efficiency_vector_host & 1 \\
\hline contact_rate_host_host & 0 \\
\hline transmission_efficiency_host_host & 0 \\
\hline mean_inoculum_host & $1.00 \mathrm{E}+02$ \\
\hline mean_inoculum_vector & $1.00 \mathrm{E}+00$ \\
\hline recovery_rate_host & $1.00 \mathrm{E}-01$ \\
\hline
\end{tabular}




\begin{tabular}{|c|c|}
\hline recovery_rate_vector & $1.00 \mathrm{E}-01$ \\
\hline lethality_rate_host & 0 \\
\hline lethality_rate_vector & 0 \\
\hline recombine_in_host & 0 \\
\hline recombine_in_vector & $1.00 \mathrm{E}-04$ \\
\hline num_crossover_host & 0 \\
\hline num_crossover_vector & 1 \\
\hline mutate_in_host & $1.00 \mathrm{E}-06$ \\
\hline mutate_in_vector & 0 \\
\hline death_rate_host & 0 \\
\hline death_rate_vector & 0 \\
\hline birth_rate_host & 0 \\
\hline birth_rate_vector & 0 \\
\hline vertical_transmission_host & 0 \\
\hline vertical_transmission_vector & 0 \\
\hline inherit_protection_host & 0 \\
\hline inherit_protection_vector & 0 \\
\hline protection_upon_recovery_host & None \\
\hline protection_upon_recovery_vector & None \\
\hline
\end{tabular}

*For a detailed description of each parameter, consult the documentation at https://github.com/pablocarderam/opqua\#newsetup

Table S3: Parameters used for two-strain compartment model

\begin{tabular}{|l|l|l}
\hline Parameter name & Description & Value
\end{tabular}


bioRxiv preprint doi: https://doi.org/10.1101/2021.12.16.473045; this version posted December 17, 2021. The copyright holder for this preprint (which was not certified by peer review) is the author/funder, who has granted bioRxiv a license to display the preprint in perpetuity. It is made available under aCC-BY 4.0 International license.

\begin{tabular}{|l|l|l|}
\hline$\delta$ & host recovery rate & $1.00 \mathrm{E}-01^{*}$ \\
\hline$\beta$ & host contact rate & $1.10 \mathrm{E}-01^{*}$ \\
\hline$\varphi$ & $\begin{array}{l}\text { probability of wild-type } \\
\text { pathogens outcompeting } \\
\text { mutants in intra-host } \\
\text { competition }\end{array}$ & $1 /(2-0.1)^{*}$ \\
\hline$n_{i}$ & size of inoculum & 1 \\
\hline$\mu_{1}$ & $\begin{array}{l}\text { mutation rate from wild-type } \\
\text { to mutant }\end{array}$ & $\begin{array}{l}5.00 \mathrm{E}-02 \\
\text { mutation rate from mutant to }\end{array}$ \\
\hline$\mu_{2}$ & total population size & $5.00 \mathrm{E}+02$ \\
\hline$N$ & &
\end{tabular}

*Parameters vary according to simulation 